NIST Technical Note 2106

\title{
Comparing Instruments
}

James Yen

Dennis Leber

Leticia Pibida

This publication is available free of charge from:

https://doi.org/10.6028/NIST.TN.2106 


\title{
Comparing Instruments
}

\author{
James Yen \\ Dennis Leber \\ Statistical Engineering Division \\ Information Technology Laboratory \\ Leticia Pibida \\ Radiation Physics Division \\ Physical Measurement Laboratory
}

This publication is available free of charge from:

https://doi.org/10.6028/NIST.TN.2106

September 2020

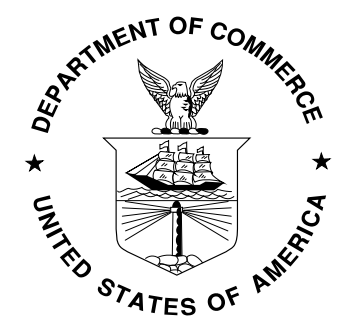

U.S. Department of Commerce Wilbur L. Ross, Jr., Secretary

National Institute of Standards and Technology Walter Copan, NIST Director and Undersecretary of Commerce for Standards and Technology 
Certain commercial entities, equipment, or materials may be identified in this document in order to describe an experimental procedure or concept adequately. Such identification is not intended to imply recommendation or endorsement by the National Institute of Standards and Technology, nor is it intended to imply that the entities, materials, or equipment are necessarily the best available for the purpose.

National Institute of Standards and Technology Technical Note 2106 Natl. Inst. Stand. Technol. Tech. Note 2106, 57 pages (September 2020) CODEN: NTNOEF

This publication is available free of charge from: https://doi.org/10.6028/NIST.TN.2106 


\begin{abstract}
This document details methods to compare instrument performance. Comparison methods for instruments outputting binary (0-1) responses as well as for instruments outputting continuous numeric responses are shown, first for two instruments and then for multiple instruments. Hypothesis tests and confidence intervals for instrument differences are demonstrated. Several nonparametric procedures are shown along with reasons why they might be needed. Finally, equivalence testing is demonstrated for those cases where testing for equivalence rather than for differences is indicated.
\end{abstract}

\title{
Keywords
}

Analysis of variance (ANOVA); binomial test response; confidence interval; continuous test response; equivalence testing; hypothesis test; nonparametric tests; normal approximation; Student's $t$ test. 


\section{Table of Contents}

$1 \quad$ Statistical preliminaries $\quad 7$

1.1 Hypothesis tests 7

1.2 Confidence intervals 8

1.3 Normal approximation tests ( $z$ tests) 9

2 Binary data $\quad 10$

2.1 Comparing two instruments with binary response 10

2.2 Comparing multiple instruments with binary response 17

3 Continuous measurements 22

3.1 Comparing two instruments with continuous data 24

3.2 Comparing multiple instruments with continuous data 32

3.3 Comparing instrument variances with continuous data 35

$4 \quad$ Non-normal data 38

4.1 Diagnosis and repair 38

4.2 Nonparametric methods 42

$5 \quad$ Proving equivalence $\quad 47$

5.1 Null hypothesis and indifference zone $\quad 47$

$5.2 \quad$ Hypothesis test 48

5.3 Example 50

5.4 Confidence interval representation 51

5.5 Power and sample size $\quad 51$

5.6 Other applications 55

6 References $\quad 56$ 


\section{List of Tables}

Table 1 Sample sizes needed for each group to satisfy several values of $\alpha$ and $\beta$, for some combinations of $P_{1}$ and $P_{2}$

Table 2 List of paired data 28

Table 3 List of instrument measurements with ranks 43

\section{List of Figures}

Figure 1 A standard normal distribution, shown with its two-sided critical points (-1.645 and 1.645) for significance level $\alpha=0.10$. If the $z$ statistic is greater than 1.645 or smaller than -1.645 , then the null hypothesis of no instrument differences is rejected.

Figure 2 A chi-square distribution with 5 degrees of freedom, shown with its critical point (11.07) for significance level $\alpha=0.05$. If the $\chi 2$ statistic is greater than the critical point, then the null hypothesis of no instrument differences is rejected.

Figure 3 This set of graphs are schematics of some different ways that the measurements of two instruments can compare with each other. In the two graphs on the left, the two instruments have the same measurement means, while in the graphs on the right, the two instruments have different measurement means. In the graphs on the top row, the two instruments have the same measurement variances, while in the graphs on the bottom row the two instruments have different measurement variances.

Figure 4 Histogram and normal probability plot of a normally distributed sample of distance measurements.

Figure 5 The histogram and normal probability plot show that this sample of distance measurements is not normally distributed.

Figure 6 The histogram and normal probability plot of the transformed data show that the transformed data approaches approximate normality.

Figure 7 Combined one-sided $95 \%$ confidence intervals for the difference in the preand post-test response. The indifference zone $\left[\delta_{L}, \delta_{U}\right]$ is displayed by the dashed lines.

Figure 8 Power curve for an ideal equivalence test with indifference zone $\left[\delta_{\llcorner}, \delta_{\mathrm{U}}\right]=$ [-4.575,4.575].

Figure 9 Power curves for several equivalence tests of varying sample sizes, $\mathrm{n}_{\mathrm{A}}=\mathrm{n}_{\mathrm{B}}$, each with a maximum type I error probability a $\alpha=0.05$, indifference zone $\left[\delta_{L}, \delta_{\cup}\right]=[-4.575,4.575]$, and $\sigma$ estimated as 2.494 .

Figure 10 Power curves for several equivalence tests of varying sample sizes, $\mathrm{n}_{\mathrm{A}}=\mathrm{n}_{\mathrm{B}}$, each with a maximum type I error probability a $\alpha=0.05$, indifference zone $\left[\delta_{L}, \delta_{U}\right]=[-4.575,4.575]$, and $\sigma$ estimated as 2.494 


\section{Comparing Instruments}

Often, experimenters wish to compare the performances of two or more instruments. For example: what is the difference between how well two detectors perform, or is there any significant differences in the instrument response between a group of several instruments?

\section{Statistical Preliminaries}

This document will begin with brief primers about hypothesis tests and confidence intervals, as those tools will be used repeatedly in this document. There will be an additional primer about using the normal approximation to create a $z$ test, which is a type of hypothesis test used repeatedly in this document.

\subsection{Hypothesis tests}

In this document we describe several hypothesis tests for comparing instrument performance. NIST Technical Note 2045 [1], the NIST/SEMATECH e-Handbook [2], and Mendenhall and Sincich [3] all give an introduction to hypothesis testing. However, since the focus of this document is the comparison of instrument performance rather than, say, confirming performance thresholds, the terminology of the hypothesis tests will be geared toward that application. In this document, the null hypothesis will usually have the form:

$H_{0}$ : All instruments perform equally well.

The alternative hypothesis will have the form:

$H_{A}$ : Not all instruments perform equally well.

What is called in the statistical literature a type I error occurs when the hypothesis test result leads us to conclude that instruments perform differently when they are not actually different. Hypothesis tests can be specified so that the probability of a type I error is bounded above by a number $\alpha$. Such a hypothesis is said to have significance level equal to $\alpha$. The most commonly used value of $\alpha$ is 0.05 , although $0.0,0.10$, and 0.20 values are also used depending on the type of instrument that is being tested and its use. These significance levels are used to weigh the risk associated with a type I error.

For our hypothesis tests, what we call a type II error occurs when there really are differences between the instruments, but the hypothesis test fails to reject the null hypothesis. The probability of such an error is usually denoted by $\beta$. The quantity $1-\beta$ is called the power of a test, which is the probability of rejecting the null hypothesis when there is an actual difference. The power of a hypothesis test is not a single number but depends on the magnitude of such an actual difference; a large difference between instrument will be much easier to detect than a small difference.

The power function of a hypothesis test is the power of the test as a function of the underlying difference. 
There are trade-offs between type I and type II errors. To carry it to the most extreme, a hypothesis test that never rejects the null hypothesis will have zero type I errors, but have no power to detect any significant differences, thus leading to unacceptably high probability of type II errors.

\subsubsection{P-values}

When one does a hypothesis test using statistical or computational software, the output usually contains a $p$-value. (Except in certain unusual cases, a $p$-value will not be available without the use of statistical software or statistical functions in computational software.) The $p$-value is used to indicate a probability that is calculated after the collected data are analysed. The $p$-value is the probability of obtaining a statistic as extreme or more extreme than what actually occurred, given that the null hypothesis is actually true. In this document on instrument comparison, the $p$-value can usually be interpreted as the probability of measuring a disparity as large or larger than that seen, under the assumption that the instruments are actually equivalent. If the p-value is smaller than $\alpha,(p<\alpha)$, then the null hypothesis is rejected by the test with significance level $\alpha$. For instance, if the $p$-value $=0.04$, then the null hypothesis would be rejected at a significance level $\alpha=0.05$, but not at a significance level $\alpha=0.01$. The $p$-value indicates the smallest significance level for which the null hypothesis would be rejected; thus, the smallness of a $p$-value can be seen as an indicator of the weight of evidence against the null hypothesis.

There is a growing attitude in the scientific and statistical communities that one should not rely solely on hypothesis tests and associated $p$-values to draw conclusions. One should also examine other procedures, especially statistical intervals, which are described in the next section, as well as always doing an exploratory data analysis that includes looking at plots of the data.

\subsection{Confidence intervals}

A hypothesis test gives no information other than whether the null hypothesis of "no difference between the instruments" should be rejected. It does not specify which instrument performed better, the magnitude of the difference, and how practically significant the difference is. However, a statistical interval, often in the form of a confidence interval, can address those very issues, at least in part. See Hahn and Meeker [4] for a fuller discussion of confidence intervals and other intervals. In this document, the intervals will usually be those of performance differences between instruments. In the examples, the performance will be measured as a probability of detection or as a continuous measurement of performance such as the limit of detection of an instrument, or the magnitude of a signal measured by an instrument. The confidence interval for a difference includes values for the difference that are plausible given the data. The length of a confidence interval depends on the specified confidence level. For example, a procedure with a $90 \%$ confidence level should include the correct value $90 \%$ of the time. The higher confidence level that is desired, the larger that confidence interval must be to attain that confidence level. Thus, a $99 \%$ confidence interval is longer than a $95 \%$ confidence interval, which is in turn longer than a $90 \%$ confidence interval. 
Often there is a correspondence between confidence intervals and hypothesis testing as follows: If a 100 $\times(1-\alpha) \%$ confidence interval for the difference contains zero, then that corresponds to the null hypothesis of "no difference" not being rejected by a corresponding hypothesis test with significance level $\alpha$. Conversely, if the $100 \times(1-\alpha) \%$ confidence interval does not contain zero, that corresponds to the null hypothesis being rejected by the corresponding hypothesis test with significance level $\alpha$.

\subsection{Normal approximation tests ( $z$ tests)}

In various places in this document, we will provide hypothesis tests and procedures that rely on a normal distribution approximation. Due to a theorem from mathematical statistics called the Central Limit Theorem, statistics that are averages of a large number of independent, identically distributed random variables with finite mean and variance approximately follow a Normal distribution, also known as a Gaussian distribution. Suppose that a random variable $x$ follows a Normal distribution with mean $\mu$ and variance $\sigma^{2}$ (and standard deviation $\sigma$ ), denoted by $N(\mu$ ,$\left.\sigma^{2}\right)$. Then, $x$ can be standardized by subtracting the mean and dividing by the standard deviation to $z=(x-\mu) / \sigma$, which has a $N(0,1)$ distribution, known as a standard normal distribution. In practice, $\mu$ and $\sigma$ are not known in advance and in the $z$ statistic formula will be replaced by estimates of those parameters.

A $z$ test involves a statistic $z$ that should approximately follow a standard normal distribution if the Null Hypothesis is true. If $|z|$ is very large, then the Null Hypothesis becomes less plausible. More exactly, the $p$-value for a hypothesis test result is defined as the probability of obtaining a result just as extreme as the observed result if the null hypothesis were true. Since we will be doing comparison tests to see if quantities are the same or different, most of the tests in this document will be two-sided in that given an observed statistic $z$, the $p$-value will be of the form $\mathrm{P}(|Z| \geq|z|)$, where $Z$ is the standard normal variate.

Unfortunately, the cumulative distribution function $P(z<Z)$ of a standard normal variate, usually denoted $\Phi(Z)$, cannot be written in closed form. However, there are tables of the percentile points of the standard normal distribution in most statistical textbooks as well as in many online resources. In particular, for the two-sided hypothesis tests used repeatedly in this document, it is useful to know the two-sided critical points of the standard normal distribution are 1.28 for significance level $\alpha=0.20,1.645$ for significance level $\alpha=0.10,1.96$ for $\boldsymbol{\alpha}=\mathbf{0 . 0 5}$, and 2.576 for $\boldsymbol{\alpha}=\mathbf{0 . 0 1}$. Most statistical and computational software packages possess a function that outputs $\Phi(Z)$ for any real value of $Z$ (as well as its inverse function $\left.\Phi^{-1}(p)\right)$ ). 


\section{Binary data}

\subsection{Comparing two instruments with binary response}

We will first explore the case of comparing two instruments having binary response. As an example, suppose that there are two detectors that register a "present/not present" response to a radioactive source. For the purposes of this section, suppose that during the measurement, a radioactive source is present so that detecting it would be considered a success, and not detecting would be a failure. Suppose there are $n_{1}$ trials for Instrument 1 and $n_{2}$ trials for Instrument 2 . The number of detections by Instrument 1 would be $x_{1}$, with the resulting proportion of success being $p_{1}=x_{1} / n_{1}$; for Instrument 2 , there would be $x_{2}$ successes out of $n_{2}$ trials with the resulting proportion $p_{2}=x_{2} / n_{2}$. The sample proportions $\boldsymbol{p}_{1}$ and $\boldsymbol{p}_{2}$ are our best estimates of the true proportions $P_{1}$ and $P_{2}$, respectively.

The number of successes for an instrument is modeled by a Binomial distribution modeling the number of successes in a set of independent Bernoulli trials; refer to NIST Technical Note 2045 [1], Ross [5] or most statistics textbooks for more background and justification for these models.

\subsubsection{Testing for differences between proportions}

\subsubsection{Large samples: $z$-test}

For relatively large sample sizes, we can create a test statistic that is approximately distributed as a standard normal variate under the null hypothesis. If the null hypothesis $H_{0}$ of "no difference between instruments" is true, let $\bar{p}=\frac{x_{1}+x_{2}}{n_{1}+n_{2}}$ be the proportion of successes in the combined sample. Define

$$
Z=\frac{p_{1}-p_{2}}{\sqrt{\bar{p}(1-\bar{p})\left(\frac{1}{n_{1}}+\frac{1}{n_{2}}\right)}}
$$

Under the null hypothesis $H_{0}$ of "no difference between instruments", with relatively large samples sizes, $z$ is approximately normal with mean 0 and variance 1 . We can apply the previous section on normal approximation tests to this $z$ statistic; the null hypothesis $H_{0}$ of "no difference between instruments" is rejected if the statistic $z$ is too large or too small to be consistent with belonging to the standard normal distribution. At the $\alpha$ significance level, that translates to rejecting the null hypothesis if $z$ falls outside the middle $100(1-\alpha / 2)$ percent of the standard normal distribution. The figure below demonstrates that situation for significance level $\alpha=0.10$; the area beneath the standard normal density curve between the two-sided critical points -1.645 and 1.645 has area $1-\alpha=0.90$. The null hypothesis of no difference is rejected if 
Standard Normal dist. with two-sided critical points for significance level 0.10

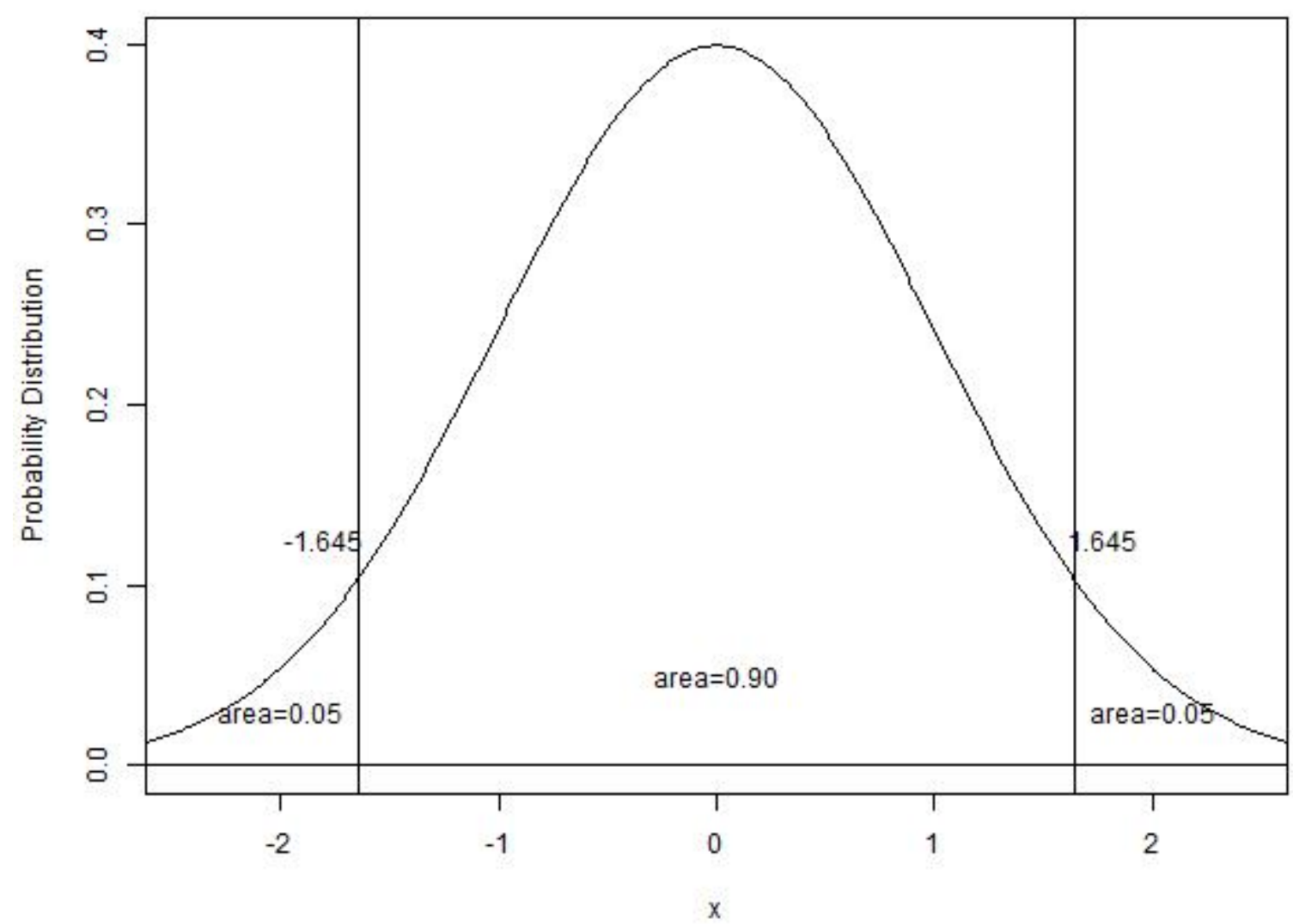

Figure 1: A standard normal distribution, shown with its two-sided critical points (-1.645 and 1.645) for significance level $\alpha=0.10$. If the $z$ statistic is greater than 1.645 or smaller than -1.645, then the null hypothesis of no instrument differences is rejected.

the $z$ statistic $z<-1.645$ or $z>1.645$.

To tell if the sample sizes are large enough, one can plug in the values of $n_{1}, n_{2}, p_{1}$, and $p_{2}$ into a formula provided by Mendenhall and Sincich [3]. They state that if the sample sizes $n_{1}$ and $n_{2}$ are large enough so that the intervals

$$
p_{1} \pm 2 \sqrt{\frac{p_{1}\left(1-p_{1}\right)}{n_{1}}} \text { and } p_{2} \pm 2 \sqrt{\frac{p_{2}\left(1-p_{2}\right)}{n_{2}}}
$$

do not contain 0 or 1 , then the normal approximation is reasonably accurate. Otherwise, if the sample sizes are small enough that the above intervals contain 0 or 1 , then the use of a nonparametric test such as Fisher's Exact Test (Section 2.1.1.2) is indicated.

Example: Suppose that we are trying to test the null hypothesis $H_{0}$ of "no difference between Instrument 1 and Instrument 2 " at a significance level $\alpha=0.10$. An experiment is performed 
where Instrument 1 successfully detects a source 14 times out of 20 trials, while Instrument 2 detects a source 10 times out of 20 trials. Thus we have $p_{1}=\frac{14}{20}=0.7$, and $p_{2}=\frac{10}{20}=0.5$, and $\bar{p}=\frac{14+10}{20+20}=0.6$. First, checking on the sample size criterion, we find that

$p_{1} \pm 2 \sqrt{\frac{p_{1}\left(1-p_{1}\right)}{n_{1}}}=0.7 \pm 2 \sqrt{\frac{0.7(1-0.7)}{20}}=0.7 \pm 0.2=(0.5,0.9)$ and

$p_{2} \pm 2 \sqrt{\frac{p_{2}\left(1-p_{2}\right)}{n_{2}}}=0.5 \pm \sqrt{\frac{0.5(1-.5)}{20}}=0.5 \pm 0.22=(0.28,0.72)$

Neither interval contains 0 or 1 , so the normal approximation should be valid.

The $z$ statistic is

$z=(0.7-05) / \sqrt{0.6(1-0.6)\left(\frac{1}{20}+\frac{1}{20}\right)}=0.2 / \sqrt{0.024}=1.291$. The magnitude of $z$ is smaller than 1.645, which is the two-sided standard normal critical point for significance level $\alpha=0.10$; therefore, the null hypothesis of no performance difference between instruments is not rejected at this significance level. Using the normal cumulative distribution in statistical software gives the additional information that the $p$-value of $z=1.291$ is $0.19>0.10$; since the $p$-value is larger than the significance level $\alpha=0.10$, the null hypothesis is not rejected.

\subsubsection{Fisher's Exact Test}

When sample sizes are too small for the normal approximation tests to be appropriate, an alternative is a nonparametric test such as Fisher's Exact Test [2]. Suppose that the results of a two-instrument test can be tabulated in a $2 \times 2$ contingency table as follows:

\begin{tabular}{|l|c|c|c|}
\hline & Successes & Failures & Column Totals \\
\hline Instrument 1 & $A$ & $B$ & $A+B$ \\
\hline Instrument 2 & $C$ & $D$ & $C+D$ \\
\hline Row Totals & $A+C$ & $B+D$ & $N=A+B+C+D$ \\
\hline
\end{tabular}

Fisher's Test enumerates how compatible the experimental results are with the null hypothesis. Under the null hypothesis of no real performance difference between the instruments, whatever differences did occur are due to chance. If we fix the marginal totals in the table and presume that the real success rate of both instruments is $(A+C) / N$, then the probability of each frequency in the contingency table is given by the hypergeometric distribution [5]:

$p=\frac{\left(\begin{array}{c}A+C \\ A\end{array}\right)\left(\begin{array}{c}B+D \\ B\end{array}\right)}{\left(\begin{array}{c}N \\ A+B\end{array}\right)}$, where $\left(\begin{array}{c}m \\ k\end{array}\right)=\frac{m !}{k !(m-k) !}$ is the number of combinations of $k$ objects that can be chosen from a collection of $m$. Since we are focused on comparing instruments, the relevant $p$ value is the two-sided $p$-value, being the total probability of possible outcomes of equal or 
greater instrument difference given fixed marginal totals. How those outcomes are tabulated is best explained using an example (below).

\section{Example}

Suppose that for an experiment testing two instruments, only 5 independent responses are available for each instrument. It is desired to have significance level $\alpha=0.05$. Suppose that Instrument 1 is successful in 4 of 5 trials, while Instrument 2 is successful in only 1 of 5 trials.

\begin{tabular}{|l|c|c|c|}
\hline & Successes & Failures & Column Totals \\
\hline Instrument 1 & 4 & 1 & 5 \\
\hline Instrument 2 & 1 & 4 & 5 \\
\hline Row Totals & 5 & 5 & 10 \\
\hline
\end{tabular}

Suppose that the recorded row and column totals are fixed, so that each instrument has 5 trials, and there is a total of 5 successes and 5 failures among these 10 total trials. A result showing the same performance difference between instruments as the actual experimental result has

probability $\frac{\left(\begin{array}{l}5 \\ 4\end{array}\right)\left(\begin{array}{l}5 \\ 1\end{array}\right)}{\left(\begin{array}{c}10 \\ 5\end{array}\right)}=\frac{25}{252}=0.099$.

The only cases that are more extreme than the actual results in opposing the null hypothesis, while retaining the same row and column totals, are the cases where one instrument has 5 successes and the other instrument has 0 successes. Each of these cases has probability

$\frac{\left(\begin{array}{l}5 \\ 5\end{array}\right)\left(\begin{array}{l}5 \\ 0\end{array}\right)}{\left(\begin{array}{c}10 \\ 5\end{array}\right)}=\frac{1}{252}=0.004$. Since each case could have either Instrument 1 or Instrument 2 having the more successes, the relevant two-sided $p$-value is $2 \times 0.099+2 \times 0.004=0.206$, so the result is not significant at the $\alpha=0.05$ level. Note that if there are only 5 observations for each instrument, the only experimental result that would reject the null hypothesis at the significance level $\alpha=0.05$ is the most extreme possible difference result of one instrument being successful in all 5 trials and the other instrument failing in all 5 trials (in which case the resulting $p$-value is $2 \times 0.004=0.008$ ). This highlights how having a small sample size limits the extent of statistical conclusions one can make, as well as the limitations of Fisher's Test. The $p$-values for Fisher's Exact Test can be laborious to calculate by hand for larger sample sizes and more moderate probabilities, but then these are the cases that are ideal for the $z$-test. Modern statistical software should make both tests easy to perform.

Regardless of which hypothesis test is utilized, users should perform the confidence interval described in the next section to further understand the nature of any differences.

\subsubsection{Confidence intervals of difference between proportions}

The hypothesis tests described in the previous sections give no information other than whether the null hypothesis of "no difference between the instruments" should be rejected. However, a 
statistical interval can address at least in part which instrument performed better, the magnitude of the difference, and how practically significant the difference is.

Let $z_{1-\frac{\alpha}{2}}$ be the $100(1-\alpha / 2)$ percentile point of the standard normal distribution. Then a widely used 100(1- $\alpha) \%$ level confidence interval for $P_{1}-P_{2}$ based on a normal approximation is:

$$
\left(p_{1}-p_{2}\right) \pm z_{1-\frac{\alpha}{2}} \sqrt{\frac{p_{1}\left(1-p_{1}\right)}{n_{1}}+\frac{p_{2}\left(1-p_{2}\right)}{n_{2}}}
$$

Unfortunately, the interval shown in Equation (2.3), which is also known as a Wald-type interval, has been shown not to achieve its desired coverage of the true difference in many cases. Agresti and Caffo [6] propose a modification of the Wald interval as follows: Produce a "modified" pseudo-data set by adding one success and one failure to each instrument's results and plug the statistics from the resulting pseudo-data set into Equation (2.3). In more detail, let the "modified" sample proportions be $\tilde{p}_{1}=\frac{x_{1}+1}{n_{1}+2}$, and $\tilde{p}_{2}=\frac{x_{2}+1}{n_{2}+2}$. Then a 100(1- $\left.\alpha\right) \%$ level confidence interval for $P_{1}-P_{2}$ with good performance qualities is:

$$
\left(\tilde{p}_{1}-\tilde{p}_{2}\right) \pm z_{1-\frac{\alpha}{2}} \sqrt{\frac{\tilde{p}_{1}\left(1-\tilde{p}_{1}\right)}{n_{1}+2}+\frac{\tilde{p}_{2}\left(1-\tilde{p}_{2}\right)}{n_{2}+2}} .
$$

Agresti and Caffo do not claim that this interval satisfies any theoretical optimality criteria; rather, they demonstrate from extensive simulation studies that it attains the proper coverage probabilities for virtually any choice of $n_{1}, n_{2}, P_{1}$, and $P_{2}$ that they examined.

This interval does not have a one-to-one correspondence with the normal approximation-based $z$ test hypothesis testing procedure described in the previous section. In this case, whether this confidence interval contains or does not contain zero does not correspond exactly to that hypothesis test accepting or rejecting, respectively, the null hypothesis of equal proportions. Each procedure was chosen to satisfy its particular purpose, although they will agree in the vast majority of cases. Agresti and Caffo [6] do not advocate using the interval (2.4) as an implicit hypothesis test instead of the $z$-test in (2.1) because doing so would be needlessly conservative in cases where the hypothetically common proportion of success $P_{1}=P_{2}$ in the null hypothesis is close to 0 or to 1 .

\section{Example.}

Let us return to our previous example of the two instruments, where Instrument 1 successfully detects a source 14 times out of 20 trials, while Instrument 2 detects a source 10 times out of 20 trials. Adding pseudo-observations of one success and one failure to each instrument's data produces "modified" sample proportions $\tilde{p}_{1}=\frac{15}{22}=0.68$, and $\tilde{p}_{2}=\frac{11}{22}=0.5$. Using the formula in (2.4), a $90 \%$ confidence interval for the difference $P_{1}-P_{2}$ is 


$$
(0.68-0.5) \pm z_{1-\frac{0.1}{2}} \sqrt{\frac{0.68(1-0.68)}{22}+\frac{0.5(1-0.5)}{22}}
$$

$=0.18 \pm 1.645(0.146)=0.18 \pm 0.24=(-0.06,0.42)$.

Note that this confidence interval contains zero at the $90 \%$ confidence level. However, we do see that the range of plausible values for the difference $P_{1}-P_{2}$ includes numbers as large as 0.42 on the positive end, but only extends to -0.06 on the negative end. Thus, it is much more plausible for $P_{1}$ to be a little larger than $P_{2}$, rather than the reverse, as would be expected given $p_{1}$ was larger than $p_{2}$.

\subsubsection{Sample Size Requirements}

\subsubsection{Sample Sizes for testing equality of proportion}

In terms of practical significance, it may be desired to specify how many trials are needed to detect a significant difference at a given significance level. For this to make sense, what must be specified first is the minimum difference the test is required to discover, as well as the minimum power and maximum Type I error needed for the Hypothesis test. We will follow the treatment of Chapter 3 of Fleiss [7]. In a problem of this type, one instrument has a relatively known success rate of $P_{1}$, and a comparison study with a new instrument is worth doing only if it is able to detect a difference between the old instrument and a new instrument with rate $P_{2}$.

Suppose we need such a test to have significance level $\alpha$ and power $1-\beta$, for a desired $\alpha$ and $\beta$. We will assume equal sample sizes for both instruments.

Let $\bar{P}=\left(P_{1}+P_{2}\right) / 2$. Let

$$
n^{\prime}=\frac{\left(z_{1-\frac{\alpha}{2}} \sqrt{2 \bar{P}(1-\bar{P})}-z_{\beta} \sqrt{P_{1}\left(1-P_{1}\right)+P_{2}\left(1-P_{2}\right)}\right)^{2}}{\left(P_{2}-P_{1}\right)^{2}}
$$

Then $n^{\prime}$ is the same size needed for each sample.

According to Fleiss, there are studies that say the above formula underestimates the needed sample size needed to achieve the desired power. This should be especially relevant when a continuity correction is utilized in the test statistic (it is not in this document). He lists a formula that uses a continuity correction in the formula to adjust the needed sample size, with an accompanying close approximation that we list here:

$$
n^{\prime \prime}=n^{\prime}+\frac{2}{\left|P_{2}-P_{1}\right|}
$$

\section{Example}


Refer to the previous example again. Let us presume the sample proportions were the actual population proportions, i.e. $P_{1}=0.7$ and $P_{2}=0.5$. Suppose we desired a hypothesis test with significance level $\alpha=0.10$ and power $(1-\beta)=0.75$. According to the formula above, the sample size for each instrument needed is $n^{\prime}=64$. If we wanted to even be more conservative, the augmented formula gives a sample size of $n^{\prime \prime}=74$. It is no wonder that our hypothesis test in the first example failed to reject the null hypothesis, as the actual sample of 20 is much smaller.

Note that if we drastically lowered the needed power of the test (1- $\beta$ ) to a mere 0.50 and weakened the significance level to $\alpha=0.20$, then a sample size of $n^{\prime}=20$ is needed.

The table below shows the sample sizes needed, showing both $n^{\prime}$ (Formula (2.5) above) and $n^{\prime \prime}$ (from Formula (2.6)), to satisfy several values of $\alpha$ and $\beta$, for some combinations of $P_{1}$, and $P_{2}$. All tables show how the sample size needed depends on the significance level and power required. Comparing the first two sub-tables shows how the required sample sizes can depend on the magnitudes of $P_{1}$ and $P_{2}$, even though $P_{1}-P_{2}$ is the same for both sub-tables. Finally, the third sub-table shows that the sample sizes needed can be much smaller if one instrument has a very sizeable advantage over the other.

\begin{tabular}{|c|c|c|c|c|c|}
\hline $\boldsymbol{\alpha}$ & $\mathbf{1 - \beta}$ & $\boldsymbol{P}_{\mathbf{1}}$ & $\boldsymbol{P}_{\mathbf{2}}$ & $\boldsymbol{n}^{\prime}$ & $\boldsymbol{n}^{\prime \prime}$ \\
\hline 0.10 & 0.60 & 0.7 & 0.5 & 43 & 53 \\
\hline 0.10 & 0.70 & 0.7 & 0.5 & 56 & 66 \\
\hline 0.10 & 0.80 & 0.7 & 0.5 & 73 & 83 \\
\hline 0.05 & 0.60 & 0.7 & 0.5 & 59 & 69 \\
\hline 0.05 & 0.70 & 0.7 & 0.5 & 73 & 83 \\
\hline 0.05 & 0.80 & 0.7 & 0.5 & 93 & 103 \\
\hline
\end{tabular}

\begin{tabular}{|c|c|c|c|c|c|}
\hline $\boldsymbol{\alpha}$ & $\mathbf{1 - \beta}$ & $\boldsymbol{P}_{\mathbf{1}}$ & $\boldsymbol{P}_{\mathbf{2}}$ & $\boldsymbol{n}^{\prime}$ & $\boldsymbol{n}^{\prime \prime}$ \\
\hline 0.10 & 0.60 & 0.9 & 0.7 & 29 & 39 \\
\hline 0.10 & 0.70 & 0.9 & 0.7 & 37 & 47 \\
\hline 0.10 & 0.80 & 0.9 & 0.7 & 49 & 59 \\
\hline 0.05 & 0.60 & 0.9 & 0.7 & 39 & 49 \\
\hline 0.05 & 0.70 & 0.9 & 0.7 & 49 & 59 \\
\hline 0.05 & 0.80 & 0.9 & 0.7 & 62 & 72 \\
\hline
\end{tabular}

\begin{tabular}{|c|c|c|c|c|c|}
\hline $\boldsymbol{\alpha}$ & $\mathbf{1 - \beta}$ & $\boldsymbol{P}_{\mathbf{1}}$ & $\boldsymbol{P}_{\mathbf{2}}$ & $\boldsymbol{n}^{\prime}$ & $\boldsymbol{n}^{\prime \prime}$ \\
\hline 0.10 & 0.60 & 0.95 & 0.6 & 10 & 16 \\
\hline 0.10 & 0.70 & 0.95 & 0.6 & 13 & 19 \\
\hline 0.10 & 0.80 & 0.95 & 0.6 & 17 & 22 \\
\hline 0.05 & 0.60 & 0.95 & 0.6 & 14 & 20 \\
\hline 0.05 & 0.70 & 0.95 & 0.6 & 17 & 23 \\
\hline 0.05 & 0.80 & 0.95 & 0.6 & 21 & 27 \\
\hline
\end{tabular}

Table 1: Sample sizes needed for each group to satisfy several values of $\alpha$ and $\beta$, for some combinations of $P_{1}$ and $P_{2}$. 


\subsubsection{Sample Sizes for estimating the difference of proportion}

Suppose there is a need to estimate the difference between the two instrument means $P_{1}-P_{2}$ to within a margin of $H$ with probability $1-\alpha$. A reasonable magnitude for $H$ depends on the context and application of the experiment. Suppose there is prior knowledge about the approximate magnitude of $P_{1}$ and $P_{2}$. Presuming equal sample sizes, the number of trials for each sample required is [3]:

$$
n=\left(\frac{z_{1-\frac{\alpha}{2}}}{H}\right)^{2}\left[P_{1}\left(1-P_{1}\right)+P_{2}\left(1-P_{2}\right)\right]
$$

If there is no prior knowledge of the approximate magnitude of $P_{1}$ and $P_{2}$, since the sample size formula is maximized by $P_{1}=P_{2}=0.5$, then a conservative estimate of the number of trials for each sample required can be obtained plugging in $P_{1}=P_{2}=0.5$ :

$$
n=\frac{1}{2}\left(\frac{z-\frac{\alpha}{2}}{H}\right)^{2}
$$

If $P_{1}$ and $P_{2}$ are much different from 0.5 , then this sample size may be much larger than needed.

\section{Example.}

Refer again to the example used in the previous sections. Suppose we had prior knowledge that the two instrument means were approximately $P_{1}=0.7$ and $P_{2}=0.5$. Suppose we needed to estimate the difference between the two instrument means $P_{1}-P_{2}$ to within a margin of 0.2 with probability 0.90 . Then the number of trials for each sample required is:

$$
\left.n=\left(\frac{1.645}{0.2}\right)^{2}[0.7(1-0.7)+) .5(1-0.5)\right]=67.64(0.46)=31.1 \approx 31 .
$$

We round $n=31.1$ to $n=31$ trials needed for each sample.

Suppose that we had no prior knowledge about $P_{1}$ and $P_{2}$, and used $P_{1}=P_{2}=0.5$ as a conservative procedure. Since the estimates we used earlier are relatively close to, or even equal to 0.5 , the resulting number $n=67.64(0.5)=33.8$, which we round to $n=34$, is not very different.

\subsection{Comparing multiple instruments with binary response 2.2.1 Chi-square test for equality of proportions}

Suppose that there are $k>2$ instruments that when tested yield Detect/No Detect binary responses. Using the same notation as the previous section, suppose that instrument $i$ has $x_{i}$ 
successes in $n_{i}$ trials, for a sample proportion of $p_{i}=x_{i} / n_{i}$, for $i=1, \ldots, k$. How do we compare these proportions?

The first step in the analysis is to test whether all instruments have the same probability of success. Here, the null hypothesis has the form:

$H_{0}:$ All instruments have the same proportion of success.

The alternative hypothesis is:

$H_{A}$ : Not all the instruments have the same proportion of success.

The test used is an example of a chi-square goodness of fit test on a contingency table [2,8]. If all proportions are the same, this common proportion can be estimated by pooling all the trials:

$$
\bar{p}=\sum_{i=1}^{k} x_{i} / \sum_{i=1}^{k} n_{i}
$$

Under the null hypothesis, the expected number of successes for the $i^{\text {th }}$ instrument is $s_{i}=$ $\boldsymbol{n}_{\boldsymbol{i}} \boldsymbol{p}_{\boldsymbol{i}}$, with corresponding expected number of failures $\boldsymbol{r}_{\boldsymbol{i}}=\boldsymbol{n}_{\boldsymbol{i}}\left(\mathbf{1}-\boldsymbol{p}_{\boldsymbol{i}}\right)$. Our test statistic is the sum of the squared deviation of the numbers of successes and failures each from its expected number, and each normalized by the expected number:

$$
\chi^{2}=\sum_{i=1}^{k} \frac{\left(x_{i}-s_{i}\right)^{2}}{s_{i}}+\sum_{i=1}^{k} \frac{\left(n_{i}-x_{i}-r_{i}\right)^{2}}{r_{i}} .
$$

Another way to write the same formula that may be more familiar to those experienced with contingency table tests is:

$$
\chi^{2}=\sum_{\text {all cells }} \frac{\left(f_{o}-f_{e}\right)^{2}}{f_{e}},
$$

Here $f_{e}$ is the expected frequency, and $f_{o}$ is the observed frequency of each particular cell out of the $2 k$ number of cells in a contingency table.

Given a significance level of $\alpha, H_{0}$ is rejected if the test statistic $\chi^{2}$ is larger than the $100(1-\alpha)$ percentile of the chi-squared distribution with $\boldsymbol{k}$ - $\mathbf{1}$ degrees of freedom. Unlike the hypothesis tests previously discussed in this document, this is a one-sided test in that only the area under the right tail of the reference distribution is the rejection region of the test. The plot below depicts a possible chi-square distribution (with 5 degrees of freedom); the critical point for that distribution (11.07) is that point where $95 \%$ of the area under the density curve lies to the left, and $5 \%$ of the area to the curve lies to the right. If the $\chi^{2}$ statistic is greater than 11.07 , then the null hypothesis of no instrument differences is rejected. Tables of critical values are available in most statistics textbooks as well as many online resources. Alternatively, one can use a statistical software to compute a $p$-value. 
Note: It is well-known that for the case of 2 instruments, this $\chi^{2}$ test is equivalent to the $z$-test comparing two proportions shown earlier in Section 2.1.1.1.

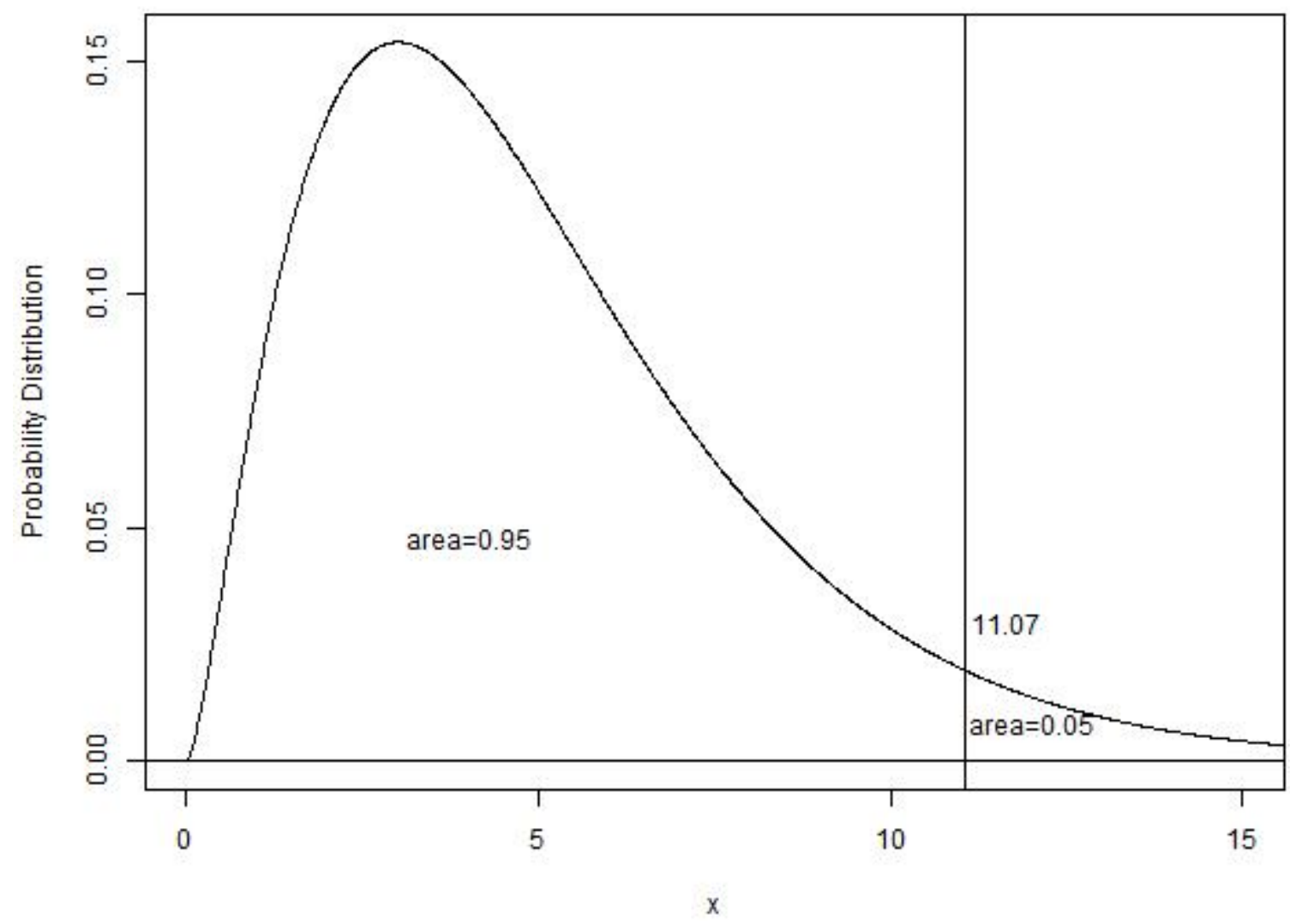

Figure 2: A chi-square distribution with 5 degrees of freedom, shown with its critical point (11.07) for significance level $\alpha=0.05$. If the $\chi^{2}$ statistic is greater than the critical point, then the null hypothesis of no instrument differences is rejected.

\section{Example}

Refer to our previous example involving two instruments. Suppose that in addition to the two instruments there was a third instrument that had 6 successes in 20 trials.

\begin{tabular}{|l|c|c|c|c|}
\hline Instrument & $\mathbf{1}$ & $\mathbf{2}$ & $\mathbf{3}$ & Row totals \\
\hline Successes & 14 & 10 & 6 & 30 \\
\hline Failures & 6 & 10 & 14 & 30 \\
\hline Column Totals & 20 & 20 & 20 & 60 \\
\hline
\end{tabular}


For this example, $\bar{p}=\frac{14+10+6}{60}=\frac{1}{2}$, and since each instrument had 20 trials, the expected number of successes for each instrument under the null hypothesis is 10 . The expected number of failures for each instrument under the null hypothesis is also 10. So, the test statistic for this example is

$$
\begin{gathered}
\chi^{2}=\frac{\left[(14-10)^{2}+(10-10)^{2}+(6-10)^{2}+(6-10)^{2}+(10-10)^{2}+(14-10)^{2}\right]}{10} \\
=6.4 .
\end{gathered}
$$

The reference distribution is the chi-square distribution with $k-1=2$ degrees of freedom. The $\chi^{2}$ statistic of 6.4 is larger than the critical point of 5.99 for significance level $\alpha=0.05$, so the null hypothesis of "all instruments being the same" is rejected. Statistical software shows that the $p$ value of 6.4 is 0.04 .

\subsubsection{Comparing Multiple proportions: Marascuilo procedure}

If running a multiple proportions contingency table hypothesis test leads you to conclude that not all instruments perform equally, it still does not inform you which instruments are better or worse. The instruments can be ranked by their respective proportions of success, but that does not indicate which proportions are significantly different. The Marascuilo procedure [2] is a way to simultaneously test the differences between all pairs of instruments.

Once again suppose that there are $k$ instruments, and that instrument $i$ has $x_{i}$ successes in $n_{i}$ trials, for a sample proportion of $p_{i}=x_{i} / n_{i}$, for $i=1, \ldots, k$.

For every pair of sample proportions, compute the absolute value of the difference $\left|p_{i}-p_{j}\right|$ for every $i \neq j$ in $1, \ldots, k$. This will be the test statistic for that pair. There will be $k(k-1) / 2$ different proportion differences.

Next for every pair of $i \neq j$, for a chosen overall significance level $\alpha$, the critical value of that test statistic will be

$$
r_{i j}=\sqrt{\chi_{1-\alpha, k-1}^{2}} \sqrt{\frac{p_{i}\left(1-p_{i}\right)}{n_{i}}+\frac{p_{j}\left(1-p_{j}\right)}{n_{j}}}
$$

Here $\chi_{1-\alpha, k-1}^{2}$ is the $100(1-\alpha)$ percentile point of a $c h i$-squared distribution with $k$ - 1 degrees of freedom. As before the numerical value of $\chi_{1-\alpha, k-1}^{2}$ can be found in the literature, online, or using a statistical software.

For each of the $k(k-1) / 2$ pairs of proportions, if $\left|p_{i}-p_{j}\right|>r_{i j}$, then those two proportions are significantly different. 


\section{Example}

Returning to the same example used in the contingency table test, there are three instruments that have 14, 10, and 6 successes out of 20 trials for each experiment. Thus, $p_{1}=0.7, p_{2}=0.5$, and $p_{3}=0.3$. There are $k(k-1) / 2=3$ possible pair differences.

A significance level of $\alpha=0.05$ will be used. Then $\chi_{1-\alpha, k-1}^{2}=\chi_{0.95,2}^{2}=5.99$.

- For Instruments 1 and $2,\left|p_{1}-p_{2}\right|=0.2<r_{12}=\sqrt{5.99} \sqrt{\frac{0.7(1-0.7)}{20}+\frac{0.5(1-0.5)}{20}}=0.37$, so Instruments 1 and 2 are not significantly different.

- For Instruments 1 and $3,\left|p_{1}-p_{3}\right|=0.4>r_{13}=\sqrt{5.99} \sqrt{\frac{0.7(1-0.7)}{20}+\frac{0.3(1-0.3)}{20}}=0.35$, so Instruments 1 and 3 are significantly different.

- For Instruments 2 and $3,\left|p_{2}-p_{3}\right|=0.2<r_{23}=\sqrt{5.99} \sqrt{\frac{0.5(1-0.5)}{20}+\frac{0.3(1-0.3)}{20}}=0.37$, so Instruments 2 and 3 are not significantly different. 


\section{Continuous measurements}

The binary nature of the data discussed earlier can sometimes make it problematic to find definitive answers with small sample sizes. If there is continuous data available, it can be much more informative than simple counts of $(0 / 1)$ trials.

As a hypothetical example, suppose that instead of (Detect/No Detect) responses from an instrument from a fixed distance from a source, there are multiple trials of the following experimental procedure: a source and a detector are gradually moved closer together until the instrument detects the source, with the detection distance recorded. Here we presume the instrument will always eventually detect the source, otherwise the result can be denoted as zero or some previously agreed to quantity. In practice, such numerical measurements are never truly continuous due to the limited resolution of measurement, and in this scenario, it would be practical to move the detector and instrument closer in steps rather than as a continuous process.

Another example of continuous measurements would be the time till detection of the source by the detector. (This test would be more likely for a chemical detector or if a radiation detector integrates the signal until there are enough counts above background to be detected.)

The increased complexity of continuous data over binary data brings an accompanying complexity in how instruments can be compared. Figure 3 contains some schematics of how instrument performances can differ. The upper left plot depicts hypothetical data of an experiment where the measurements taken from Instrument 1 and Instrument 2 have the same mean and variability. The upper right plot depicts an experiment where the data from the two instruments have the same variability, but different means. In the lower left plot, the measurements taken on both Instrument 1 and Instrument 2 are centered on the same mean, but those of Instrument 2 are much more variable. The lower right plot shows a situation where the measurements from Instrument 2 are both larger on the average and more variable than those of Instrument 1. This section on comparing instruments that produce continuous measurements will concentrate first on location-based methods for comparing instruments based on analysis of their mean measurements, before moving to methods for comparing instruments by examining the respective variances of the instrument measurements. 

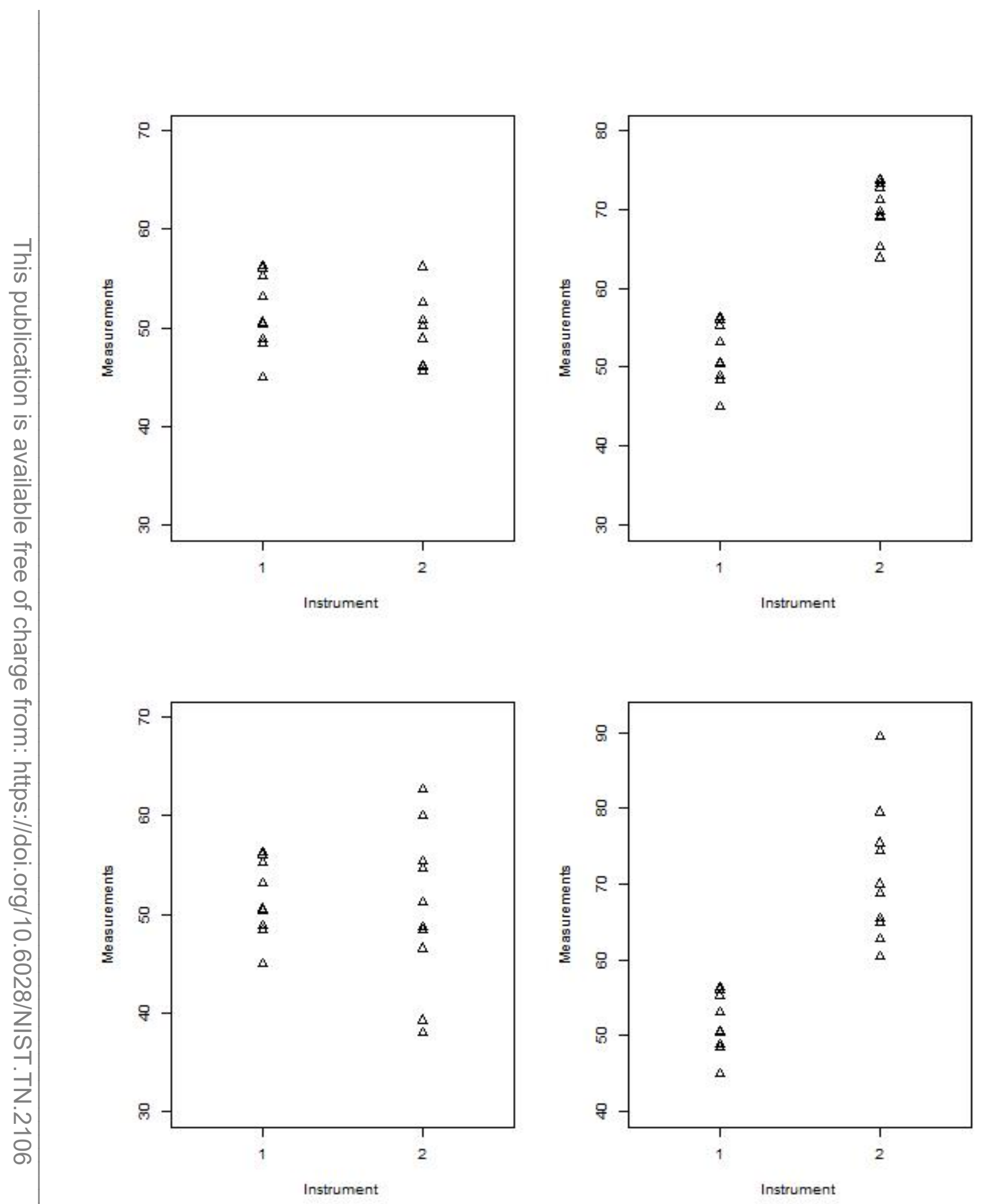

Figure 3: This set of graphs are schematics of some different ways that the measurements of two instruments can compare with each other. In the two graphs on the left, the two instruments have the same measurement means, while in the graphs on the right, the two instruments have different measurement means. In the graphs on the top row, the two instruments have the same measurement variances, while in the graphs on the bottom row the two instruments have different measurement variances. 


\subsection{Comparing two instruments with continuous measurements}

Suppose we want to compare two instruments where the data are in the form of numeric measurements. For instance, they may be from multiple trials of the distance till first detection of the source, as described in the previous section. For the rest of this section we have the following notation for the observations:

Suppose for Instrument 1 , there are $m \geq 2$ measurements denoted as: $x_{1}, \ldots, x_{m}$.

Suppose for Instrument 2, there are $n \geq 2$ measurements denoted as: $y_{1}, \ldots, y_{n}$.

Let $\bar{x}=\frac{1}{m} \sum_{i=1}^{m} x_{i}$ be the mean of the measurements for Instrument 1 .

Let $\bar{y}=\frac{1}{n} \sum_{j=1}^{n} y_{j}$ be the mean of the measurements for Instrument 2 .

Let $s_{x}^{2}=\frac{1}{m-1} \sum_{i=1}^{m}\left(x_{i}-\bar{x}\right)^{2}$ be the sample variance of the measurements for Instrument 1 .

Let $s_{y}^{2}=\frac{1}{n-1} \sum_{j=1}^{n}\left(y_{j}-\bar{y}\right)^{2}$ be the sample variance of the measurements for Instrument 2 .

\subsubsection{Hypothesis tests for comparing two instruments}

When comparing two instruments where the data are in the form of numeric measurements, the simplest way to compare is to test whether they have the same mean. The classical test is the two-sample Student's $t$-test, of which we describe two variations here.

Suppose that the measurements $x_{1} \ldots, x_{m}$ come from a distribution with mean $\mu_{1}$, and the measurements $y_{1}, \ldots, y_{n}$ come from a distribution with mean $\mu_{2}$.

For the $t$-test, the null and alternative hypotheses will be:

$$
\begin{aligned}
& H_{0}: \mu_{1}=\mu_{2} \\
& H_{A}: \mu_{1} \neq \mu_{2}
\end{aligned}
$$

\subsubsection{Student's $t$-test: Equal variance case.}

Suppose it is presumed or verified (see Section 3.3 on comparing variances) that the measurements for both instruments have approximately the same variance. Then we can use a pooled estimator for the variance that pools the sample variances from both instruments:

$$
s_{p}^{2}=\frac{(m-1) s_{x}^{2}+(n-1) s_{y}^{2}}{m+n-2} .
$$

Our test statistic is

$$
T=\frac{\bar{x}-\bar{y}}{s_{p} \sqrt{\frac{1}{m}+\frac{1}{n}}} .
$$


For a given significance level $\alpha$, reject the null hypothesis that the two instruments are equal if

$$
|T|>t_{1-\alpha / 2, v},
$$

Where $t_{1-\alpha / 2, v}$ is the $100(1-\alpha)$ percentile of the Student's $t$ distribution with $v$ degrees of freedom. For this test, $v=m+n-2$.

Tables of $t$-test critical values are widely available in any statistics textbook, as well as online resources such as the NIST-Sematech handbook [2].

\subsubsection{Student's $t$-test: Unequal variance case.}

If the two variances are presumed or tested not to be equal, or if there is doubt about the equality of the variances, then one should not utilize the pooled variance estimate. In that case the preferred form of a $t$-test statistic is

$$
T=\frac{\bar{x}-\bar{y}}{\sqrt{\frac{s_{x}^{2}}{m}+\frac{s_{y}^{2}}{n}}}
$$

For a given significance level $\alpha$, reject the null hypothesis that the two instruments are equal if

$$
|T|>t_{1-\alpha / 2, v},
$$

Here the degrees of freedom used is given by the Welch-Satterthwaite formula [2]:

$$
v=\frac{\left(\frac{s_{x}^{2}}{m}+\frac{s_{y}^{2}}{n}\right)^{2}}{\left(\frac{s_{x}^{2}}{m}\right)^{2} /(m-1)+\left(\frac{s_{y}^{2}}{n}\right)^{2} /(n-1)}
$$

The calculated estimate of $v$ can be rounded to the nearest or to the next lowest integer; alternatively, many statistical software packages have $t$-distribution functions that do not require the degrees of freedom to be integers.

\subsubsection{Student's $t$-test Example:}

Suppose for each of two instruments, a sample of 15 measurements of the distance till detection was measured in $\mathrm{cm}$.

The measurements for Instrument $1\left(x_{1}, \ldots, x_{15}\right)$ :

919510710510285889210199102851149195 
For Instrument 2, the measurements are $\left(y_{1}, \ldots, y_{15}\right)$ :

\section{7}

Let's proceed with a Student's $t$-test with significance level $\alpha=0.05$.

Thus, $m=n=15, \quad \bar{x}=96.8, \quad \bar{y}=88.8, s_{x}^{2}=71.17$, and $s_{y}^{2}=112.6$.

\section{Equal Variance test}

Let us first presume that the variances of the distribution are equal. Then the pooled estimate of variance is

$$
s_{p}^{2}=\frac{(15-1) 71.17+(15-1) 112.6}{15+15-2}=91.885
$$

Thus, $s_{p}=\sqrt{91.885}=9.586$.

Our test statistic is

$$
T=\frac{96.8-88.8}{9.586 \sqrt{\frac{1}{15}+\frac{1}{15}}}=\frac{8.0}{3.5}=2.29
$$

The critical point $t_{1-\frac{\alpha}{2}, m+n-2}=t_{0.975,28}$ is $2.05<|2.29|$, so the null hypothesis of equal mean is rejected.

\section{Unequal Variance test}

In the unequal variance $t$-test, the denominator of the $T$ statistic is

$$
\sqrt{\frac{s_{x}^{2}}{m}+\frac{s_{y}^{2}}{n}}=\sqrt{\frac{71.17}{15}+\frac{112.6}{15}}=3.5
$$

The approximate degrees of freedom is estimated by the Satterthwaite formula. Plugging numbers in the formula gives

$$
v=\frac{150.1}{5.633}=26.6
$$

which we can round down to $v=26$. Thus,

$$
|T|=\frac{8.0}{3.5}=2.29>t_{0.975,27}=2.06
$$


so the null hypothesis is rejected in this case as well.

\subsubsection{Student's $t$-test: Paired tests}

There can be situations where data measurements from different instruments can be paired. For instance, suppose there were a series of different configurations of radioactive sources, and the distance till detection was measured simultaneously for two instruments for each configuration. Suppose the measurements for all instruments vary depending on the configuration, but that the differences between instruments are consistent across configurations and can be logically grouped into a sample. It is then more efficient and powerful to reduce the data to a single sample of paired differences $d_{1}=x_{1}-y_{1}, \ldots, d_{k}=x_{k}-y_{k}$.

The paired Student's $t$ test essentially takes the differences $d_{1}, \ldots, d_{k}$ and performs a one sample $t$-test of whether the differences have mean zero.

Let $\bar{d}=\sum_{i=1}^{k} d_{i} / k$ be the mean of the sample of differences, and $s_{d}^{2}=\frac{1}{(k-1)} \sum_{i=1}^{k}\left(d_{i}-\bar{d}\right)^{2}$ be the sample variance of the differences. The test statistic is

$$
T=\frac{\bar{d}}{s_{d} / \sqrt{k}} .
$$

For a selected significance level $\alpha$, the null hypothesis is rejected if

$$
|T|>t_{1-\frac{\alpha}{2}, k-1}
$$

\section{Example of paired $t$-test:}

Suppose that a performance measure for Instrument 1 and for Instrument 2 is measured simultaneously on $k=20$ different trials. Presume that it makes sense to look at the pooled set of paired differences. The measurements $x_{i}$ for Instrument 1 , the measurements $y_{i}$ for Instrument 2 , as well as the differences $d_{i}=x_{i}-y_{i}$ are listed in Table 2 below. Calculations show

$\bar{d}=6.6$, and $s_{d}^{2}=49$. Thus, $T=\frac{6.6}{7 / \sqrt{20}}=4.2$.

With a significance level of $\alpha=0.05$, $t_{1-\frac{\alpha}{2}, k-1}=t_{0.975,19}=2.1$, so the null hypothesis of no difference between the instruments is rejected. In fact, statistical software shows that the associated $p$-value is 0.0005 , which should be very strong evidence for a difference in instruments.

Note that if one applied either of the unpaired t-tests described above with this data, the pvalue would be around 0.265 , so a significant difference between instruments would not be detected. That is because the instrument difference would be hidden amidst the large 
differences between measurements for each instrument. This highlights the importance of knowing the background behind one's data and tailoring the analysis accordingly.

\begin{tabular}{|c|c|c|}
\hline $\boldsymbol{x}_{\boldsymbol{i}}$ & $\boldsymbol{y}_{\boldsymbol{i}}$ & $\boldsymbol{d}_{\boldsymbol{i}}=\boldsymbol{x}_{\boldsymbol{i}}-\boldsymbol{y}_{\boldsymbol{i}}$ \\
\hline 102 & 89 & 13 \\
\hline 67 & 60 & 7 \\
\hline 109 & 101 & 8 \\
\hline 97 & 96 & -1 \\
\hline 72 & 73 & 7 \\
\hline 46 & 39 & 13 \\
\hline 83 & 70 & -6 \\
\hline 86 & 92 & 23 \\
\hline 58 & 35 & 1 \\
\hline 65 & 64 & 6 \\
\hline 73 & 67 & 2 \\
\hline 92 & 90 & 2 \\
\hline 105 & 93 & 12 \\
\hline 96 & 84 & -3 \\
\hline 92 & 95 & 7 \\
\hline 60 & 53 & 14 \\
\hline 88 & 74 & 3 \\
\hline 78 & 75 & 12 \\
\hline 65 & 53 & 1 \\
\hline 64 & 63 & \\
\hline & & \\
\hline
\end{tabular}

Table 2: List of paired data

\subsubsection{Confidence intervals for differences between means}

As stated earlier in the section on binary results, readers are urged to proceed beyond hypothesis testing and to examine confidence intervals summarizing the difference between instruments' performance. The same data scenario will be repeated:

Suppose that the measurements $x_{1} \ldots, x_{m}$ for Instrument 1 come from a distribution with mean $\mu_{1}$, and the measurements $y_{1}, \ldots, y_{n}$ come for a distribution with mean $\mu_{2}$. It is desired to find a confidence interval for the difference $\mu_{1}-\mu_{2}$.

We will describe three different confidence intervals to use depending on the assumptions and data scenario. They will be closely linked to the three different kinds of Student's $t$ test described earlier. For the first two intervals, we again use the following quantities: 
Let $\bar{x}=\frac{1}{m} \sum_{i=1}^{m} x_{i}$ be the mean of the measurements for Instrument 1 .

Let $\bar{y}=\frac{1}{n} \sum_{j=1}^{n} y_{j}$ be the mean of the measurements for Instrument 2 .

Let $s_{x}^{2}=\frac{1}{m-1} \sum_{i=1}^{m}\left(x_{i}-\bar{x}\right)^{2}$ be the sample variance of the measurements for Instrument 1 .

Let $s_{y}^{2}=\frac{1}{n-1} \sum_{j=1}^{n}\left(y_{j}-\bar{y}\right)^{2}$ be the sample variance of the measurements for Instrument 2 .

\subsubsection{Confidence intervals for differences between means: Equal variance case}

Suppose it is presumed or verified (see Section 3.3 on comparing variances) that the measurements for both instruments have approximately the same variance. Then we can use a pooled estimator for the variance that pools both sample variances:

$$
s_{p}^{2}=\frac{(m-1) s_{x}^{2}+(n-1) s_{y}^{2}}{m+n-2} .
$$

For a given confidence level $\alpha$, the two-sided confidence interval for $\mu_{1}-\mu_{2}$ is

$$
\bar{x}-\bar{y} \pm t_{1-\frac{\alpha}{2}, m+n-2} s_{p} \sqrt{\frac{1}{m}+\frac{1}{n}}
$$

\subsubsection{Confidence intervals for differences between means: Unequal variance case:}

For a given confidence level $\alpha$, the two-sided confidence interval for $\mu_{1}-\mu_{2}$ is

$$
\bar{x}-\bar{y} \pm t_{1-\frac{\alpha}{2}, v} \sqrt{\frac{s_{x}^{2}}{m}+\frac{s_{y}^{2}}{n}}
$$

where $v$ is the effective degrees of freedom estimated by the Welch-Satterthwaite approximation $[2]$.

\section{Examples for confidence intervals for differences between means}

Refer to the data set in the $t$-test example in section 3.2. Recall that $m=n=15, \quad \bar{x}=96.8$, $\bar{y}=88.8, s_{x}^{2}=71.17, s_{y}^{2}=112.6$, and $s_{p}=9.586$. 


\section{Example: Equal variances confidence interval}

A $95 \%$ confidence interval for the difference between instrument means, assuming equal variances, is

$$
96.8-88.8 \pm t_{.975,28} 9.586 \sqrt{\frac{1}{15}+\frac{1}{15}}=8.0 \pm 7.17=(0.83,15.17) .
$$

A $95 \%$ confidence interval for the mean difference between instruments, assuming unequal variances, is

$$
96.8-88.8 \pm t_{.975,26} \sqrt{\frac{71.17}{15}+\frac{112.6}{15}}=8.0 \pm 7.19=(0.81,15.19) .
$$

The interval is slightly wider than the equal-variance interval. Note that for this example, we rounded down the Satterthwaite approximation for effective degrees of freedom $v=26.6$ to $v=26$ (this would be appropriate for those using tables of the Student's $t$ distribution). Many statistical software packages will not round $v$ because they can calculate percentiles of Student's $t$ distribution with non-integer degrees of freedom.

\subsubsection{Confidence intervals for differences between means: Paired case}

Assume that the data from the two observations are paired in a way that it makes sense to look at the sample of paired differences $d_{1}=x_{1}-y_{1}, \ldots, d_{k}=x_{k}-y_{k}$.

Let $\bar{d}=\sum_{i=1}^{k} d_{i} / k$, and $s_{d}^{2}=\frac{1}{(k-1)} \sum_{i=1}^{k}\left(d_{i}-\bar{d}\right)^{2}$.

For a given confidence level $\alpha$, the two-sided confidence interval for $\mu_{1}-\mu_{2}$ is

$$
\bar{d} \pm t_{1-\frac{\alpha}{2}, k-1} \frac{s_{d}}{\sqrt{k}} .
$$

\section{Example.}

Refer to the data contained in the table on paired $t$-tests in Section 1.3.1.3. A $95 \%$ confidence interval for the difference between instrument means is

$$
\bar{d} \pm t_{1-\frac{\alpha}{2}, k-1} \frac{s_{d}}{\sqrt{k}}=6.6 \pm \frac{7}{\sqrt{20}}=(3.32,9.88) .
$$




\subsubsection{Sample size requirements for estimating mean difference}

Suppose it is needed to estimate the difference between the two instrument means $\mu_{1}-\mu_{2}$ to within a margin of $\mathrm{H}$ with probability $1-\alpha$. Suppose also that the variances $\sigma_{1}^{2}$ and $\sigma_{2}^{2}$ are known, or at least can be estimated or approximated. Presuming equal sample sizes, the number of measurements for each sample required is:

$$
n=\left(\frac{z_{1-\frac{\alpha}{2}}}{H}\right)^{2}\left(\sigma_{1}^{2}+\sigma_{2}^{2}\right)
$$

(Mendenhall and Sincich [3]).

\section{Example.}

The variances $\sigma_{1}^{2}$ and $\sigma_{2}^{2}$ are often not known and have to be estimated from prior knowledge or approximated. Let us go back to our previous data example with the unpaired $t$-test from section 3.1.2 and presume that we had some prior knowledge that $\sigma_{1}^{2}$ is approximately 100 and $\sigma_{2}^{2}$ is approximately 120 . If it is needed to estimate the difference between the two instrument means $\mu_{1}-\mu_{2}$ to within $10 \mathrm{~cm}$ with probability 0.95 , then the number of measurements for each sample required is:

$n=\left(\frac{1.96}{10}\right)^{2}(100+120)=8.45$, which we round up to $n=9$.

Suppose there was a more stringent objective of estimating the difference between the two instrument means $\mu_{1}-\mu_{2}$ to within $5 \mathrm{~cm}$ with probability 0.95 ; then the number of measurements for each sample required is $n=\left(\frac{1.96}{5}\right)^{2}(100+120)=33.8$, which we round to $n=34$. 


\subsection{Comparing multiple instruments with continuous data 3.2.1 Hypothesis test: ANOVA test}

Suppose there are multiple instruments where the performance output is in the form of continuous numerical measurements. There will be $k$ instruments. Instrument $i$ has $n_{i}$ measurements $y_{i 1}, \ldots, y_{i n_{i}}$ that originate from a distribution with mean $\mu_{i}$. Experiment designers should know that it is advantageous for the sample sizes $n_{1}, \ldots, n_{k}$ to be equal.

A one-way Analysis-of-variance (ANOVA) hypothesis test to compare how the instruments perform will compare the means of their measurements.

The model underlying the ANOVA is that the $j^{\text {th }}$ observation from the $i^{\text {th }}$ instrument can be written as:

$$
y_{i j}=\mu+a_{i}+e_{i j} .
$$

This model decomposes each observation into three components: an overall mean $\mu$, an instrument effect $a_{i}$ (the deviation of the $i^{\text {th }}$ Instrument mean from the grand mean), and a residual $e_{i j}$. The ANOVA model presumes that the residuals are independent, approximately normally distributed with mean 0 , and have approximately the same variance for each instrument.

The null hypothesis that all instruments perform the same will be

$$
H_{0}: \mu_{1}=\cdots=\mu_{k}
$$

The alternative hypothesis is $H_{A}$ : Not all the $\mu_{i}$ are the same.

In terms of the ANOVA model, the null hypothesis $H_{0}$ states that for each instrument $a_{i}=0$.

Denote the $i^{\text {th }}$ instrument mean as $\bar{y}_{i \cdot}=\frac{1}{n_{i}} \sum_{j=1}^{n_{i}} y_{i j}$.

Let $N=\sum_{i=1}^{k} n_{i}$ be the total number of observations.

The grand mean is $\bar{y}_{. .}=\frac{1}{N} \sum_{i=1}^{k} \sum_{j=1}^{n_{i}} y_{i j}$.

The sum of squares due to Factor, which in our context is the Instrument is

$$
S S F=\sum_{i=1}^{k} n_{i}\left(\bar{y}_{i \cdot}-\bar{y}_{. .}\right)^{2}
$$

The sum of squares due to Error (residual) is

$$
S S E=\sum_{i=1}^{k} \sum_{j=1}^{n_{i}}\left(y_{i j}-\bar{y}_{i .}\right)^{2} .
$$

The mean squares are the sum of squares divided by the associated degrees of freedom:

$M S F=S S F /(k-1)$ and $M S E=S S E /(N-k)$. 
The test statistic is $F=M S F / M S E$.

Under the null hypothesis of no instrument effects, $M S F$ and $M S E$ would be essentially estimating the same quantity of variation and would follow an $F_{k-1, N-k}$ distribution, which is an $F$ distribution with $k-1$ and $N-k$ degrees of freedom. Under the alternative hypothesis, the existence of significant instrument effects should cause $M S F$ to be larger than MSE. Thus, the null hypothesis is rejected at significance level $\alpha$ if the test statistic $F$ is larger than the 100(1- $\alpha)$ percentile point of the $\boldsymbol{F}_{\boldsymbol{k}-1, N-\boldsymbol{k}}$ distribution. Critical points of the $F$ distribution for selected significance levels and various degrees of freedom are available in many statistics textbooks and online resources. Statistical software will usually provide a $p$-value associated with the result.

\section{Example}

Suppose for each of three instruments, a sample of 5 measurements of the distance till detection was measured in $\mathrm{cm}$.

The measurements for Instrument $1\left(y_{1,1}, \ldots, y_{1,5}\right)$ :

\section{9}

The measurements for Instrument $2\left(y_{2,1}, \ldots, y_{2,5}\right)$ :

\section{6}

The measurements for Instrument $3\left(y_{3,1}, \ldots, y_{3,5}\right)$ :

\section{4}

Doing the ANOVA calculations show that for the sums of squares $S S F=4954.5$ and $S S E=$ 1314.4. The relevant degrees of freedom are $k-1=(3-1)$, and $N-k=(15-3)$. Thus, the $F$-statistic is

$$
F=\frac{M S F}{M S E}=\left(\frac{S S F}{2}\right) /\left(\frac{S S E}{12}\right)=22.61 .
$$

For significance level $\alpha=0.05$, the relevant critical point of the $F_{2,12}$ distribution is 3.9 , which is much smaller than the observed $F$-statistic, so the null hypothesis of no mean instrument differences is rejected. 


\subsubsection{Multiple Comparisons}

\subsubsection{Tukey Procedure}

If the $F$-test rejects the null hypothesis of no instrument difference, it does not show how or which instrument means are different from each other. If one particular pair of instruments are singled out for comparison, then a confidence interval for that difference can be calculated. However, it is not appropriate to compute 100(1- $\alpha) \%$ intervals for all or multiple pairwise comparisons between means because the 100(1- $\alpha) \%$ confidence level will not hold for the set of comparisons.

One can use the Tukey method to estimate intervals for all pairwise mean differences and an overall confidence level of $100(1-\alpha) \%$ for the set of intervals. Suppose that there are $k$ instruments, and all the data set up, notation, and quantities are the same as in the previous section on the ANOVA test. Assume all instruments have the same sample size $n_{i}=n$, so the total number of observations is $N=k n$.

Let $M S E$ be the same mean squares as in the previous section. For a given overall significance level $\alpha$, for every pair of $\mathrm{i} \neq \mathrm{j}$, the set of simultaneous $100(1-\alpha) \%$ confidence intervals for the instrument mean differences includes

$$
\overline{y_{l .}}-\overline{y_{J .}} \pm q_{1-\alpha ; k, N-k} \sqrt{\frac{M S E}{n}}
$$

Here $q_{1-\alpha ; k, N-k}$ is the $100(1-\alpha)$ percentile point of the studentized range distribution with parameters $k$ and $N-k$. While not as well-known as the $t$ or $F$ distributions, tables of the studentized range distribution are available in many statistics textbooks and online resources. Many statistical software packages also contain a function for percentile points of the distribution for given parameters

\section{Example.}

Let's return to the same data used in the ANOVA test example in the previous section. For that data set, $\mathrm{MSE}=1314.4 / 12=109.53$, so $\sqrt{\frac{M S E}{n}}=\sqrt{\frac{109.53}{5}}=4.68$. The multiplier for a set of $95 \%$ simultaneous confidence intervals for the mean differences is $q_{0.95 ; 3,12}=3.77$, so

$q_{1-\alpha ; k, N-k} \sqrt{\frac{M S E}{n}}=3.77(4.68)=17.66$. If we denote the mean for Instrument $i$ as $\mu_{i}$, the set of intervals for the mean instrument differences are:

$$
\begin{aligned}
& \mu_{2}-\mu_{1}: 19.4 \pm 17.66=(1.74,37.06) \\
& \mu_{3}-\mu_{1}: 44.4 \pm 17.66=(26.74,62.06) \\
& \mu_{3}-\mu_{2}: 25.0 \pm 17.66=(7.34,42.66)
\end{aligned}
$$


All instrument means are significantly different from each other, with $\mu_{3}$ the largest, $\mu_{1}$ the smallest, and closest together are $\mu_{1}$ and $\mu_{2}$.

\subsubsection{Tukey-Kramer Procedure for unequal sample sizes}

The Tukey procedure in the previous section presumed equal sample sizes for each instrument. A modification called the Tukey-Kramer procedure can handle unequal sample sizes. Each interval width is calculated separately depending on the sample size.

Let $M S E$ be the same mean squares as in the previous section. For a given overall significance level $\alpha$, for every pair of $i \neq j$, the set of simultaneous confidence intervals for the instrument mean differences includes

$$
\overline{y_{l .}}-\overline{y_{J}} \pm q_{1-\alpha ; k, N-k} \sqrt{\frac{M S E}{2}} \sqrt{\frac{1}{n_{i}}+\frac{1}{n_{j}}} .
$$

This procedure is known to be conservative in that its true confidence level is larger than 1- $\alpha$ when sample sizes are unequal, meaning that the intervals are longer than they need to be to achieve a true $100(1-\alpha) \%$ confidence level for the set of simultaneous intervals.

\subsection{Comparing instrument variances with continuous data}

\subsubsection{Comparing variances from two instruments}

Recall the data scenario from Section 3.1.1 when comparing two instruments where the data are in the form of numeric measurements. Suppose that the measurements $x_{1} \ldots, x_{m}$ come from a distribution with variance $\sigma_{1}^{2}$, and the measurements $y_{1}, \ldots, y_{n}$ come from a distribution with variance $\sigma_{2}^{2}$. An $F$-test can be used to test whether the variances are equal.

For this $F$-test, the null and alternative hypotheses will be:

$$
\begin{aligned}
& H_{0}: \sigma_{1}^{2}=\sigma_{2}^{2} \\
& H_{A}: \sigma_{1}^{2} \neq \sigma_{2}^{2}
\end{aligned}
$$

Let $s_{x}^{2}=\frac{1}{m-1} \sum_{i=1}^{m}\left(x_{i}-\bar{x}\right)^{2}$ be the sample variance of the measurements for Instrument 1.

Let $s_{y}^{2}=\frac{1}{n-1} \sum_{j=1}^{n}\left(y_{j}-\bar{y}\right)^{2}$ be the sample variance of the measurements for Instrument 2 .

Then the $F$-statistic takes the form $F=s_{x}^{2} / s_{y}^{2}$.

If the significance level is $\alpha$, then the null hypothesis that the two population variances are equal is rejected if $F<F_{\alpha / 2, m-1, n-1}$ or $F>F_{1-\alpha / 2, m-1, n-1}$, where $F_{\beta, m-1, n-1}$ is the $100(\beta)$ th percentile point of the $\mathrm{F}$ distribution with $m-1$ and $n-1$ degrees of freedom. Critical points 
of the $F$ distribution for selected significance levels and degrees of freedom are available in statistical textbooks and software. Note that this is the same $F$ distribution in Section 3.2.1, although this test and its relevant test statistic is different.

\section{Example:}

We return to the example from Section 3.1.1.1. The measurements for Instrument 1 are $\left(x_{1}, \ldots, x_{15}\right)$ :

\section{5}

For Instrument 2, the measurements are $\left(y_{1}, \ldots, y_{15}\right)$ :

\section{7}

Thus, $m=n=15, s_{x}^{2}=71.17$, and $s_{y}^{2}=112.6$. The $F$-statistic $F=71.17 / 112.6=0.632$.

If the significance level is, $\alpha=0.05$, we find that the $F$-statistic is between the two critical points:

$$
F_{\alpha / 2, m-1, n-1}=F_{0.05,14,14}=0.336<F=0.632<F_{1-\alpha / 2, m-1, n-1}=F_{0.975,14,14}=2.98 .
$$

The null hypothesis of equal variances is not rejected because the null hypothesis is rejected only if the $F$-statistic is outside the interval formed by the two critical points.

This $F$-test assumes that both distributions are normally distributed. If that assumption is in doubt, one can utilize a test that is robust to assumptions. Such a test is the Levene Test, which is described in the next section.

\subsubsection{Comparing variances from multiple instruments}

The Levene test can be used to test if multiple samples come from distributions with the same variance [2]. Levene's test is more robust to assumptions than a more classical procedure like the Bartlett test, which assumes normal distributions for the data. This section describes a form of Levene's test that was proposed by Brown and Forsythe [9].

Suppose that there are $k$ samples, each from its own distribution, with $k \geq 2$. For this test, the null and alternative hypotheses will be:

$$
\begin{gathered}
H_{0}: \sigma_{1}^{2}=\sigma_{2}^{2}=\cdots=\sigma_{k}^{2} \\
H_{A}: \sigma_{i}^{2} \neq \sigma_{j}^{2} \text { for some } 1 \leq i<j \leq k
\end{gathered}
$$

Suppose that from the $k$ samples, sample $i$ has $n_{i}$ measurements $y_{i 1}, \ldots, y_{i n_{i}}$. Let $N=\sum_{i=1}^{k} n_{i}$ be the total number of observations. Define $\tilde{y}_{i}$ to be the median of the ith sample. Let

$$
z_{i j}=\left|y_{i j}-\tilde{y}_{i}\right| .
$$


Suppose $\bar{z}_{i}$. is the mean of the $z_{i j}$ for the $i^{\text {th }}$ group, and $\bar{z}$.. is the grand mean of all the $z_{i j}$. Then, the test statistic for Levene's test is

$$
W=\frac{(N-k) \sum_{i=1}^{k} n_{i}\left(\bar{z}_{i .}-\bar{z}_{. .}\right)^{2}}{(k-1) \sum_{i=1}^{k} \sum_{j=1}^{n_{i}}\left(z_{i j}-\bar{z}_{i} .\right)^{2}} .
$$

The null hypothesis of equal variances is rejected if $W>F_{1-\alpha, k-1, n-k}$, which is the $100(1-$ $\alpha$ /2) percentile point of the $F$ distribution with $k-1$ and $N-k$ degrees of freedom.

Note that the original formulation of Levene's test used the sample mean instead of the median in the definition of the $z_{i j}$. Brown and Forsythe [9] proposed modifying the test to use the median or trimmed mean instead of the mean. This section uses the median form of the test because it should be robust to non-normality while still maintaining good efficiency [2].

\section{Example}

Let us return to the example from Section 3.2.1. Suppose for each of three instruments, a sample of 5 measurements:

The measurements for Instrument $1\left(y_{1,1}, \ldots, y_{1,5}\right)$ :

6788726869

The measurements for Instrument $2\left(y_{2,1}, \ldots, y_{2,5}\right)$ :

998310210176

The measurements for Instrument $3\left(y_{3,1}, \ldots, y_{3,5}\right)$ :

108132114108124

The respective medians of the three samples are 69, 99, and 114. Thus, we have

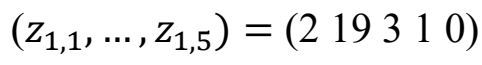

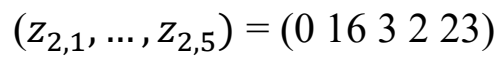

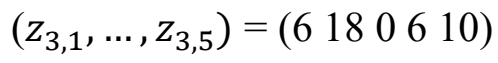

Further calculation shows that the test statistic is $W=\frac{(15-3)(40.133)}{(3-1)(836.8)}=0.288$. If the significance level is $\alpha=0.05$, then the criticial value is $F_{0.95,2,12}=3.9$. Since the test statistic $\mathrm{W}=0.288$ is far below the critical value, we cannot reject the null hypothesis of equal variances 


\section{Non-normal data}

The $t$-test and its associated confidence interval as well as ANOVA are designed to be optimal when the data follow a normal distribution (also known as a Gaussian distribution). While the $t$ test is known to be robust to Type I errors when the data deviate from normality, it always pays to graph the data to check its shape, trends, and variability.

\subsection{Diagnosis and repair}

\subsubsection{Graphical tools}

It is recommended that experimenters always plot their data. Even the most rudimentary plots that could be done with paper and pencils in the past can provide insights into the data that can go beyond summary statistics.

A histogram can be useful for checking if the data approximately follows the bell-shaped curve of the normal distribution. If available, normal probability plots, also known as qq-norm plots (or normal Q-Q plots), should also be checked. The normal probability plot is a graph that plots the ordered measurements on the vertical axis versus the median or average of the corresponding order statistics of a standard normal sample of the same size. Samples that are normally distributed should produce a normal probability plot where the points appear to be scattered close to a straight line. A normal probability plots that is far from linearity is evidence of nonnormality. Unfortunately, it is unfeasible to do a probability plot by hand; however, it is available in many statistical and worksheet programs. A histogram and a normal probability plot of a normally distributed sample is shown in Figure 4. 
Histogram of Distance

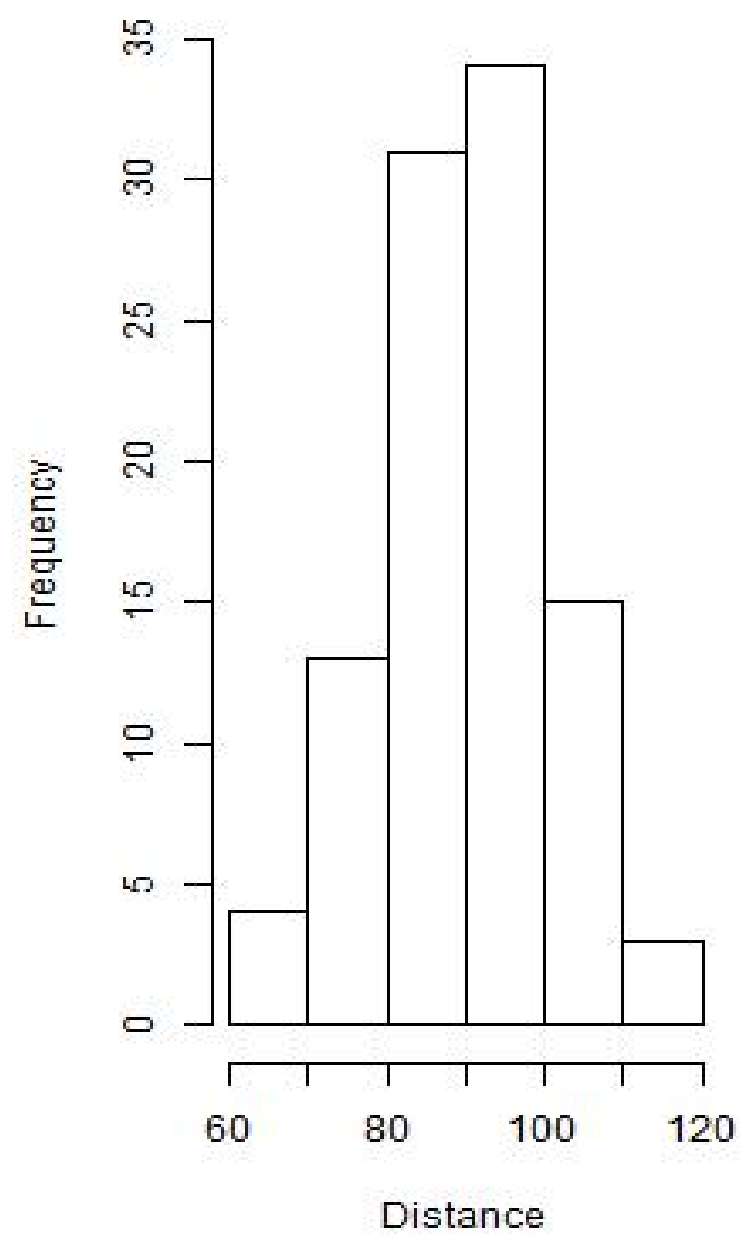

Normal Q-Q Plot

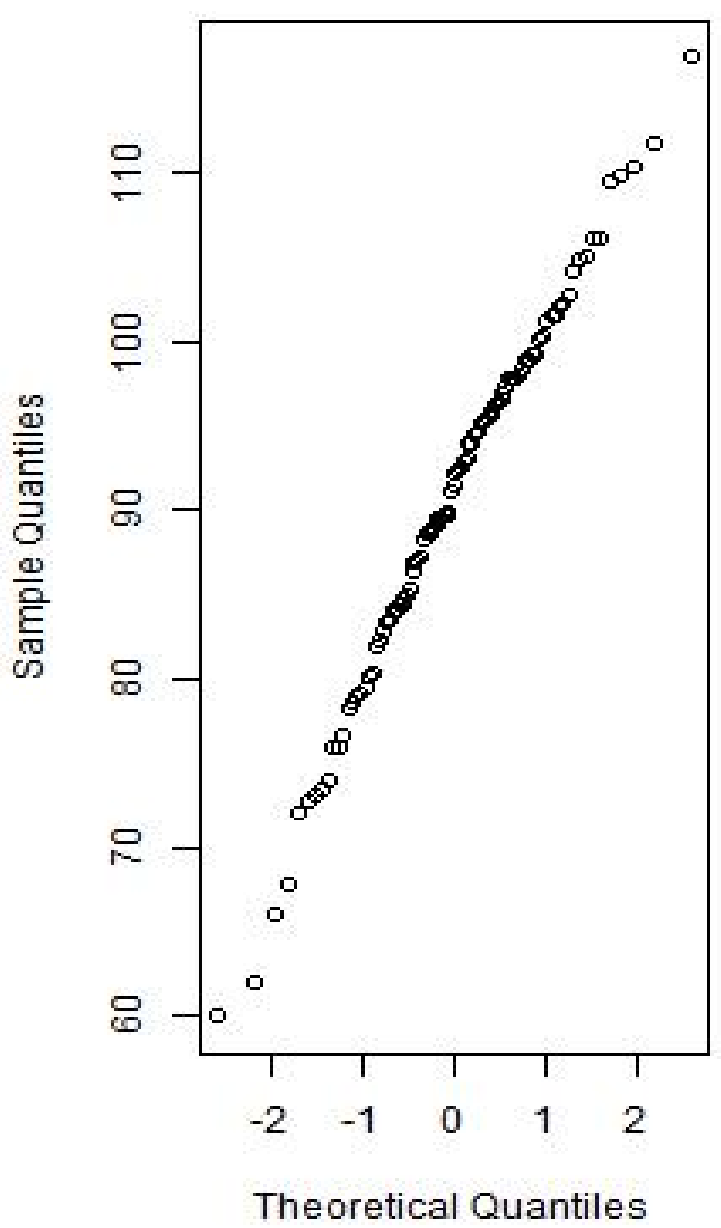

Figure 4: Histogram and Normal probability plot of a normally distributed sample of distance measurements

\subsubsection{Transformations}

It is possible that the data will be approximately normally distributed if transformed. Ideally, there will be a physical basis such a transformation. Otherwise, a particularly useful family of transformations is the Box-Cox transformation:

$$
T(X)=\frac{X^{\lambda}-1}{\lambda}
$$

Here $\lambda$ is a parameter chosen to make the data Gaussian. For $\lambda=0, T(X)=\log (X)$.

The logarithmic transformation is the most commonly used transformation for positive measurement data that are skewed to the right. 


\section{Example}

Testing of one instrument yielded 20 measurements of distance till detection (in $\mathrm{cm}$ ): 68454776048143391092382175632911101372783531975262764

The histogram and normal probability plots ( Figure 5) of this data show that it is not normally distributed.
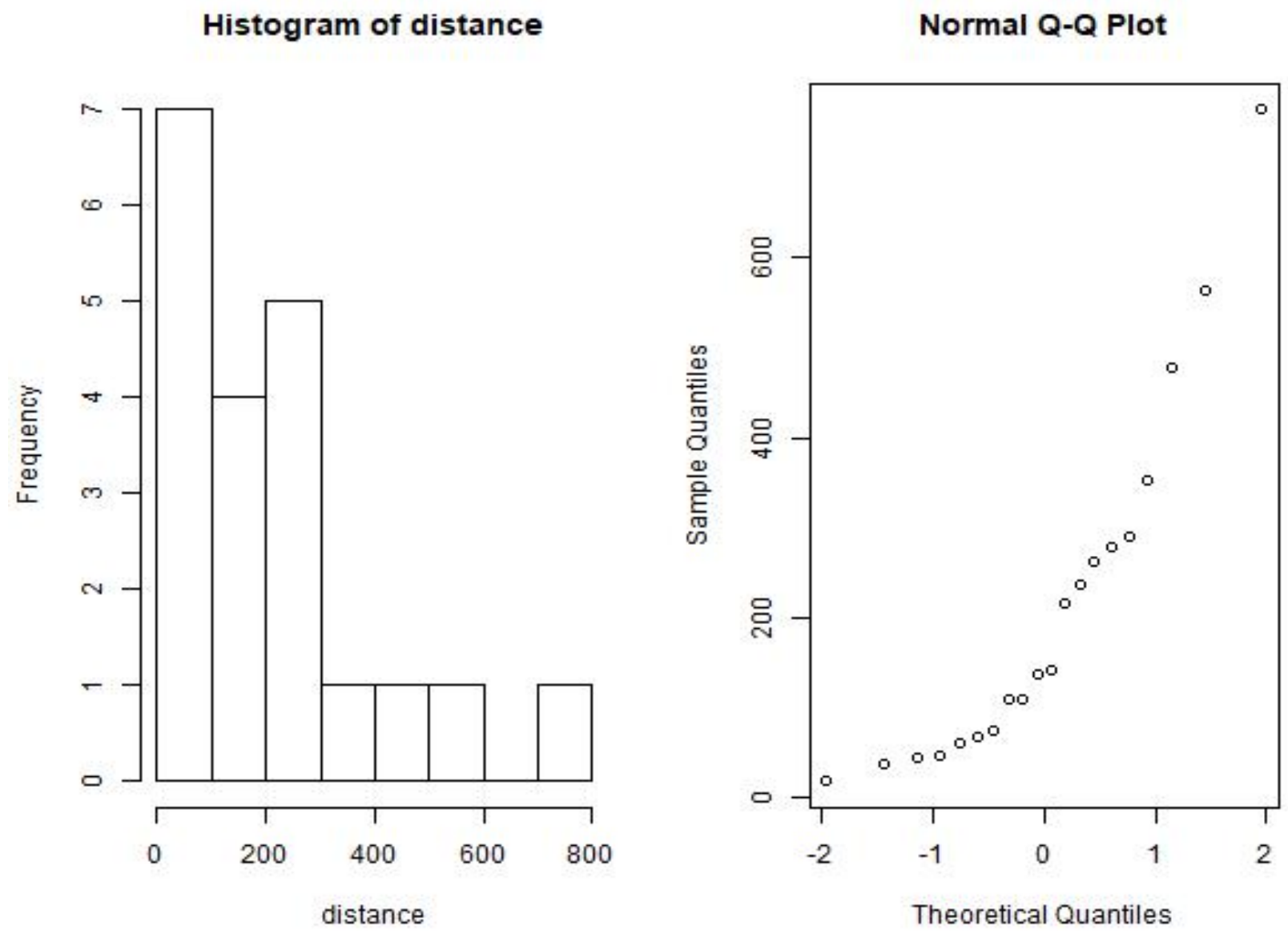

Figure 5: The histogram and normal probability plot show that this sample of distance measurements is not normally distributed.

Using a logarithmic transformation on the distance data yields:

4.223 .816 .174 .093 .874 .963 .664 .695 .475 .386 .335 .674 .704 .925 .635 .872 .944 .325 .57 6.64 
The histogram and normal probability plot of the transformed data inFigure 6 show that the transformed data approaches approximate normality.

Histogram of log.distance

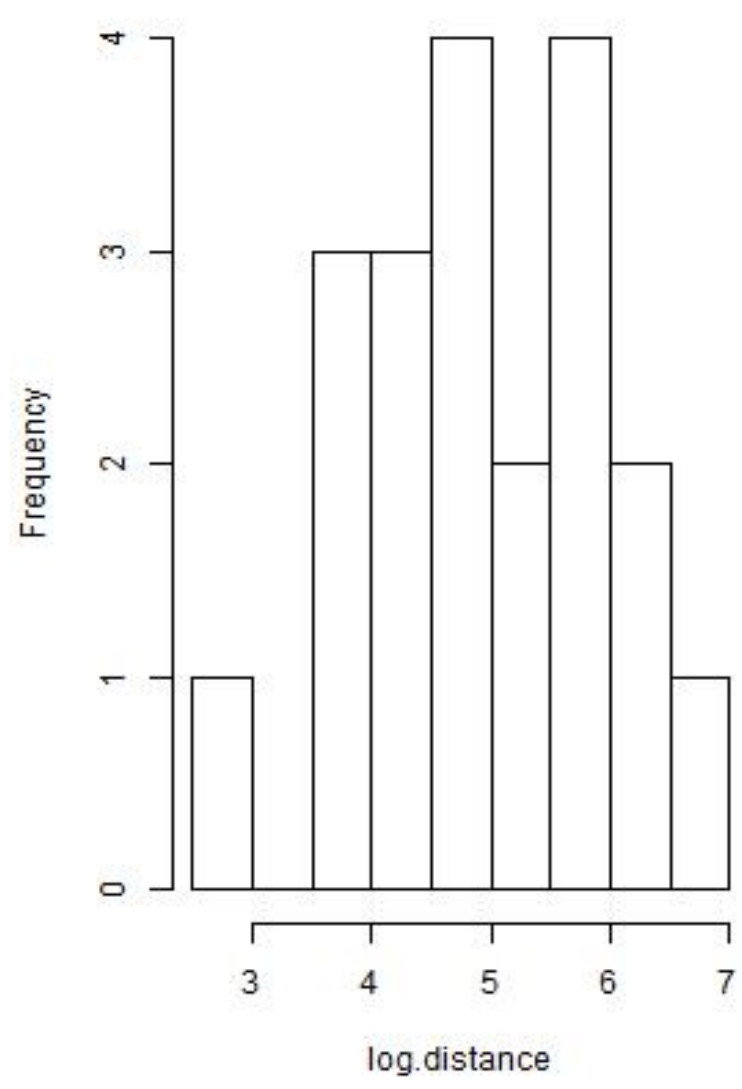

Normal Q-Q Plot

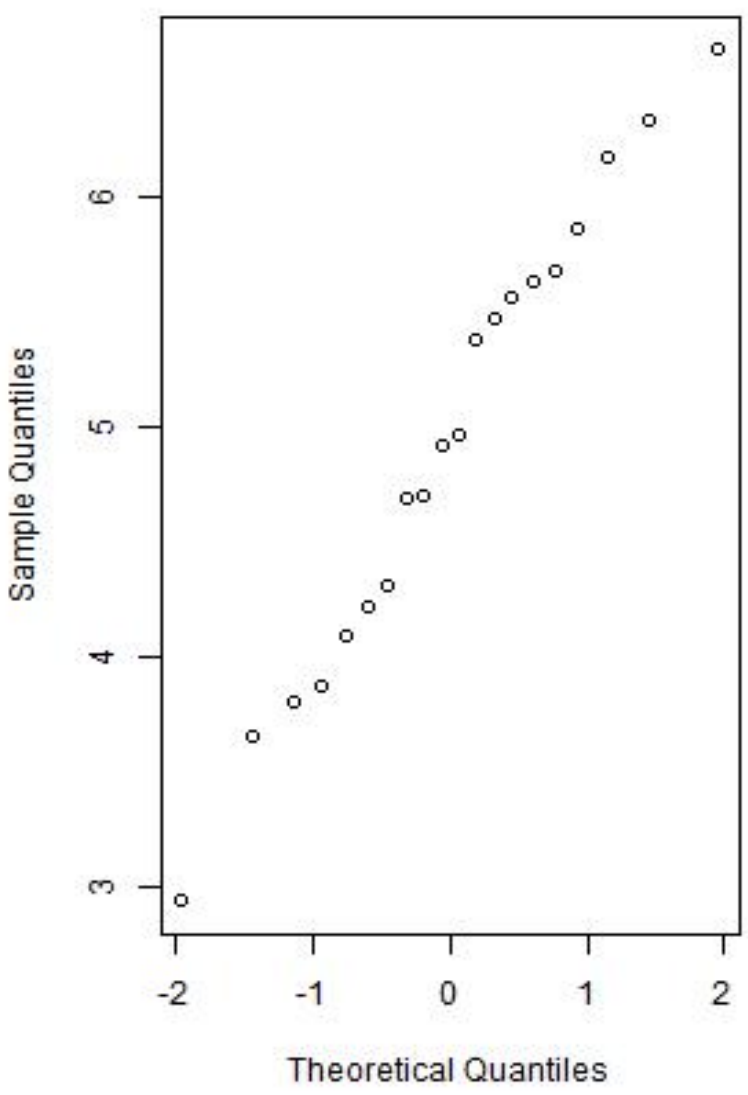

Figure 6 : The histogram and normal probability plot of the transformed data show that the transformed data approaches approximate normality.

One problem with transforming the data is the need to refer to the transformed data rather than the original data, as well as the possibility of no appropriate transformation existing for that particular data. 


\subsection{Nonparametric methods}

It may be important to use methods not beholden to any, or at least many fewer, distributional assumptions. Nonparametric methods attempt to achieve some of the same purposes as more classical procedures such as Student's $t$ test and the ANOVA F-test, but without the same required distributional assumptions. There is always a trade-off as classic methods are optimal for normally distributed data, but nonparametric methods can be much more powerful for nonnormal data. Also, nonparametric statistics tend to be more cumbersome to compute than classical statistics, but the increasing prevalence of computational and statistical software has lessened that burden considerably.

\subsubsection{Wilcoxon test for comparing two distributions}

A robust efficient alternative to the $t$-test that is not based on normal data is the Wilcoxon Rank Sum test. Note that another popular nonparametric test known as the Mann-Whitney U-test has been shown to be equivalent to the Wilcoxon test, so it will not be covered here. For the Wilcoxon test, the null hypothesis is that the two distributions are equivalent, while the alternative hypothesis is that one of the distributions is shifted to the right or the left of the other distribution [3]. The test is designed for continuous numerical data, as the presence of many ties in the combined data set (as might happen in a situation where the same number occurs many times, as in a Likert scale sample) can be problematic.

Suppose that measurements for Instrument 1 are $x_{1}, \ldots, x_{m}$, and for Instrument 2 are $y_{1}, \ldots, y_{n}$. Then rank all $m+n$ measurements in order of magnitude, with the smallest one receiving rank one and the largest one receiving rank $m+n$. Tied observations are given ranks equal to the mean of their ranks. For example, if the seventh and eighth smallest measurements are the same, both measurements are assigned the rank of 7.5. Let $\mathrm{T} 1$ be the sum of the ranks from Instrument 1 , and $\mathrm{T} 2$ be the sum of the ranks from Instrument 2 . If the null hypothesis that the two distributions are the same is true, then one would expect that the average rank from each distribution would be roughly the same; for the equal sample size case of $m=n$, we would expect $\mathrm{T} 1$ and $\mathrm{T} 2$ to be similar; in that case, if either $\mathrm{T} 1$ or $\mathrm{T} 2$ were much larger than the other rank sum, that would be evidence against the null hypothesis. In practice take the rank sum of the sample with fewer observations (if $m=n$, then take either rank sum), and compare to the appropriate critical points given $m$ and $n$ in a table of Wilcoxon Rank Sum Test critical values, which should be in many statistics textbooks and online resources. If both sample sizes are large, say at least 10, then a normal approximation test can be used. Let T1 be the rank sum of the sample with fewer measurements (making $m \leq n$ in the following formula). Let

$$
Z=\left(\mathrm{T} 1-\left[\frac{m n+m(m+1)}{2}\right]\right) / \sqrt{\frac{m n(m+n+1)}{12}}
$$

Then it is known that under the null hypothesis of identical populations, the statistic $Z$ can be approximated by a normal distribution with mean 0 and variance 1 . Therefore, the null 
hypothesis of same distributions is rejected if $Z$ is beyond the critical point in a standard normal table, i.e. for significance level $\alpha$, reject the equality of the instruments if $|Z|>z_{1-\frac{\alpha}{2}}$.

\section{Example}

Suppose for each of two instruments, a sample of 10 measurements of the distance till detection was measured in $\mathrm{cm}$.

The measurements for Instrument $1\left(x_{1}, \ldots, x_{10}\right)$ :

345338622136462873107

For Instrument 2, the measurements are $\left(y_{1}, \ldots, y_{10}\right)$ :

7257705412712861569955

It is needed to test whether the samples come from the same distribution. Neither sample appears normally distributed, so it is decided to use a Wilcoxon test. First, the two samples are combined, and each observation is assigned a rank. These ranks are listed in the following table.

\begin{tabular}{|c|c|c|}
\hline $\boldsymbol{x}_{\boldsymbol{i}}, \boldsymbol{y}_{\boldsymbol{i}}(\boldsymbol{c m})$ & Rank & Instrument \\
\hline 21 & 1 & 1 \\
\hline 28 & 2 & 1 \\
\hline 34 & 3 & 1 \\
\hline 36 & 4 & 1 \\
\hline 38 & 5 & 1 \\
\hline 46 & 6 & 1 \\
\hline 53 & 7 & 1 \\
\hline 54 & 8 & 2 \\
\hline 55 & 9 & 2 \\
\hline 56 & 10 & 2 \\
\hline 57 & 11 & 2 \\
\hline 61 & 12 & 2 \\
\hline 62 & 13 & 1 \\
\hline 70 & 14 & 2 \\
\hline 72 & 15 & 2 \\
\hline 73 & 16 & 1 \\
\hline 99 & 17 & 2 \\
\hline 107 & 18 & 1 \\
\hline 127 & 19 & 2 \\
\hline 128 & 20 & 2 \\
\hline
\end{tabular}

Table 3: List of instrument measurements with ranks. 
The sum of ranks of the Instrument 1 measurements is $\mathrm{T} 1=75$, while the sum of ranks of the Instrument 2 measurements is $\mathrm{T} 2=135$. Since both sample sizes are at least 10 , the normal approximation can be used, leading to the statistic:

$$
\begin{gathered}
Z=\left(75-\left[\frac{10(10)+10(10+1)}{2}\right] / \sqrt{\frac{10(10)(10+10+1)}{12}}\right. \\
=-\frac{30}{13.23}=-2.27 .
\end{gathered}
$$

If a significance level of $\alpha=0.05$ is used, the critical point is $z_{.975}=1.96<|-2.27|$, so the null hypothesis of no instrument difference is rejected. Using statistical software on this example will yield a $p$-value of 0.02 .

\subsubsection{Sign test for paired comparisons}

Let us go back to the situation of paired samples in Section 3.1.1.3, for which we previously used the paired $t$-test. Suppose that the assumptions of normally distributed differences do not hold. A simple nonparametric alternative to the paired $t$-test that is easy to calculate is the sign test, which tests if the median difference is significantly different from zero. For this paired situation, the sign test reduces each paired difference as either positive $(+)$ or negative $(-)$. Suppose we presume the two distributions were equal, then a $(+)$ would be equally likely as a (-). Then under this null hypothesis, if $\mathrm{S}+$ is the number out of $k$ paired differences that are positive, and $\mathrm{S}-$ is the number out of $k$ paired differences that are negative, then both $\mathrm{S}+$ and $\mathrm{S}-$ would follow a Binomial distribution with $k$ trials and probability $1 / 2$, with average value being $k / 2$. Let $\mathrm{S}=\max (\mathrm{S}+, \mathrm{S}-)$. Then, the $p$-value associated with $\mathrm{S}$ is $2 \mathrm{P}(X \geq \mathrm{S})$ if $X$ is a Binomial random variable with $k$ trials and probability $1 / 2$. For a hypothesis test with significance level $\alpha$, the null hypothesis is rejected if this $p$-value is less than $\alpha$.

The Binomial distribution with $k$ trials and $\mathrm{p}=1 / 2$ can be approximated by a Normal distribution with mean $k / 2$ and variance $k / 4$ for large $k$, say $k \geq 10$ [3]. Thus, we can again transform the test into a $z$ statistic:

$$
Z=\frac{S-\frac{k}{2}}{\sqrt{k / 4}} .
$$

The null hypothesis is rejected if $z$ is greater than the $100(1-\alpha / 2)$ th percentile point of the standard normal distribution.

\section{Example}

Refer to the dataset highlighted in the example in Section 3.1.3 on the paired $t$-test. The set of 20 paired differences between the two instrument measurements is: 
$\begin{array}{lllllllllllllllllllll}13 & 7 & 8 & 1 & -1 & 7 & 13 & -6 & 23 & 1 & 6 & 2 & 12 & 12 & -3 & 7 & 14 & 3 & 12 & 1 .\end{array}$

Note that 17 differences are positive, and 3 are negative. Suppose the significance level is $\alpha=0.05$. If $X$ is a binomial random variate with 20 trials and probability 0.5 , then the associated $p$-value is $2 \mathrm{P}(X \geq 17)=0.0026$. Thus, the null hypothesis of equal distributions (or at least equal medians) is rejected.

It may be easier for some to use the normal approximation, which produces a $z$ statistic of

$$
Z=\frac{17-\frac{20}{2}}{\sqrt{\frac{20}{4}}}=3.13
$$

which also signifies rejection of the null hypothesis.

Since much of the information in the data is not taken into account, the sign test is much less powerful and efficient than the paired t-test if the data are normal or close to it. Conversely, the sign test can be more powerful than the t-test in non-normal cases.

\subsubsection{Kruskal-Wallis procedure for testing multiple distributions}

Suppose there are $k$ instruments each yielding approximately continuous numerical measurements, with the $i^{\text {th }}$ instrument having $n_{i}$ measurements. The Kruskal-Wallis $(\mathrm{KW})$ procedure tests the null hypothesis that all $k$ samples come from the same distribution without making the distributional assumptions of ANOVA. The alternative hypothesis is that at least two of the distributions differ in location from each other. The samples do need to be random and independent. As with the Wilcoxon test, the measurements should be continuous numerical data to avoid repeated data values.

The KW procedure is similar to the Wilcoxon Test described in one of the previous sections in that it is based on ranks. Put all $N=\sum_{i=1}^{k} n_{i}$ measurements into one sample and rank them in order of magnitude, with the smallest one receiving rank one and the largest one receiving rank $N$. Tied observations are given ranks equal to the mean of their ranks. Let the sum of ranks for the $i^{\text {th }}$ instrument be $R_{i}$. If the null hypothesis is true, and if each sample size is at least 5 , then the test statistic

$$
H=\left[\frac{12}{N(N+1)} \sum_{i=1}^{k}\left(R_{i}^{2} / n_{i}\right)\right]-3(N+1)
$$

should be approximated by a chi-square distribution with $k-1$ degrees of freedom. For significance level $\alpha$, the null hypothesis is rejected if $H$ is greater than the $100(1-\alpha)$ percentile point of the $\chi_{k-1}^{2}$ distribution. 


\section{Example}

Refer back to the data set of the example in Section 3.2.1 on ANOVA. The three samples are pooled and then ranked according to magnitude as:

\begin{tabular}{|c|c|c|}
\hline $\begin{array}{c}\text { Distance } \\
\text { (cm) }\end{array}$ & Rank & Instrument \\
\hline 67 & 1 & 1 \\
\hline 68 & 2 & 1 \\
\hline 69 & 3 & 1 \\
\hline 72 & 4 & 1 \\
\hline 76 & 5 & 2 \\
\hline 83 & 6 & 2 \\
\hline 88 & 7 & 1 \\
\hline 99 & 8 & 2 \\
\hline 101 & 9 & 2 \\
\hline 102 & 10 & 2 \\
\hline 108 & 11.5 & 3 \\
\hline 108 & 11.5 & 3 \\
\hline 114 & 13 & 3 \\
\hline 124 & 14 & 3 \\
\hline 132 & 15 & 3 \\
\hline
\end{tabular}

The rank sums are: $R_{1}=17, R_{2}=38, R_{3}=65$. So the test statistic is

$$
H=\frac{12}{15(15+1)}(1191.6)-3(15+1)=11.6
$$

For significance level $\alpha=0.5$, the null hypothesis is rejected because $\mathrm{H}=11.6>\chi_{0.95: 2}^{2}=5.99$.

Statistical software shows the $p$-value is 0.003 . 


\section{Proving Equivalence}

The methods provided thus far have sought to show that the true mean performances of two (or more) instruments are different. In these traditional hypothesis test formulations, we began with the assumption that the instruments' true performances are the same and sought data-based evidence to prove that they are different. In this section we present the equivalence test (Wellek 2010), (Richter and Richter 2002) that allows for the experimenter to prove that the true mean performances of two (or more) instruments are the same.

The traditional hypothesis test formulation allows an experimenter to prove that the true mean performances of two (or more) instruments are different.

The equivalence test allows an experimenter to prove that the true mean performances of two (or more) instruments are the same.

For a practical example where an experimenter may like to prove that the true mean performances of two instruments are equivalent, we look to the American National Standards Institute's, American National Standard Performance Criteria for Alarming Personal Radiation Detectors for Homeland Security, ANSI N42.32(2016). The functionality tests of ANSI N42.32 seek to demonstrate that an instrument is unaffected by an imposed environmental, electromagnetic, or mechanical shock. That is, the experimenter would like to prove that the performance of the instrument after the imposed shock is equivalent to the performance of the instrument prior to being exposed to the shock. Though we are not strictly comparing two instruments, we can view the pre-shock instrument and the post-shock instrument as two instruments.

\subsection{Null Hypothesis and Indifference Zone}

The equivalence test differs from the traditional hypothesis test formulation on two main accounts:

1. In equivalence testing we begin with the assumption (null hypothesis) that the instruments' performances are different. We then seek data-based evidence to prove that they are the same.

2. The equivalence test only considers differences in instruments' performances that are of practical significance to the experimenter.

Recall that the hypothesis test relies on the idea of proof by contradiction. That is, we state as the null hypothesis the conjecture that is opposite of what we would like to prove. In the traditional hypothesis test formulation where we seek to identify a meaningful difference, we define the null hypothesis as the instruments' true performances being the same. If we find sufficient evidence to reject the null hypothesis, then we can confidently state that the instruments' true performances are different. Failure to reject the null hypothesis does not prove that the instruments' true performances are the same, but rather that insufficient evidence was found to support the conclusion that the instruments' true performances are different.

In equivalence testing it is our goal to prove that the true mean performances of two (or more) instruments are the same. Thus, we begin with the assumption (null hypothesis) that the 
instruments' performances are different and seek evidence to show that they are the same. When we find such evidence to support the rejection of the null hypothesis, we can then confidently state that the instruments' true performances are equivalent. Here again, failure to reject the null hypothesis is not proof the that null hypothesis is true, but simply indicates that insufficient evidence was found to support the conclusion that the instruments' true performances are equivalent.

Experimenters may find comfort in the fact that the equivalence test allows for deviations that are viewed as practically irrelevant in its definition of "the same". In developing the equivalence test, the experimenter must define the "indifference zone" where differences in the instruments' true mean performances are small enough to not be considered of practical importance in the context of the decision being made. We adopt the notation $\left[\delta_{L}, \delta_{U}\right]$ to represent the indifference zone where $\delta_{L}$ provides the lower bound of the indifference zone and $\delta_{L}$ provides the upper bound. Guidance for choosing the endpoints of the indifference zone can be found in Wellek (2010) and AndersonCook and Borror (2016).

For example, though not formulated strictly as an equivalence test, the functionality tests of ANSI N42.32 are written in such a way as to not penalize an instrument if its post-shock performance is within $15 \%$ of its pre-shock performance. If we let $\mu_{p r e}$ represent the instrument's true pre-shock

performance, then the indifference zone is $\left[-0.15 \mu_{\text {pre }}, 0.15 \mu_{\text {pre }}\right]$. That is, a difference between the pre-shock and post-shock instrument performance that is within $\pm 15 \%$ of the pre-shock performance is considered practically irrelevant.

\subsection{Hypothesis Test}

In equivalence testing we begin with the null hypothesis that the instruments' performances are different and seek evidence that allows us to reject the null hypothesis and prove that they are the same. Let $\mu_{A}$ represent the true performance of instrument $\mathrm{A}$ and $\mu_{B}$ represent the true performance of instrument B. We define "different" such that the difference in the instruments' true performances, $\mu_{A}-\mu_{B}$ falls outside of the indifference zone $\left[\delta_{L}, \delta_{U}\right]$. That is the instruments are considered different if $\mu_{A}-\mu_{B}<\delta_{L}$ or $\mu_{A}-\mu_{B}>\delta_{U}$. We see that this equates to not a single hypothesis test, but two one-sided tests (TOST) as provided in Equations (5.1) and (5.2), where $H_{0}$ and $H_{1}$ represent the null and alternative hypothesis.

$$
H_{0}: \mu_{A}-\mu_{B}<\delta_{L} \quad \text { versus } \quad H_{1}: \mu_{A}-\mu_{B} \geq \delta_{L}
$$

and,

$$
H_{0}: \mu_{A}-\mu_{B}>\delta_{U} \quad \text { versus } \quad H_{1}: \mu_{A}-\mu_{B} \leq \delta_{U}
$$

Assuming that the instruments' performances are measured using a continuous measurement, the test statistics used to evaluate these two one-sided tests are based on Student's $t$-test (see Section 3 ) and are provided in Equations (5.3) and (5.4). 


$$
\begin{aligned}
& T_{L}=\frac{\left(\bar{X}_{A}-\bar{X}_{B}\right)-\delta_{L}}{s_{p} \sqrt{\frac{1}{n_{A}}+\frac{1}{n_{B}}}} \\
& T_{U}=\frac{\left(\bar{X}_{A}-\bar{X}_{B}\right)-\delta_{U}}{s_{p} \sqrt{\frac{1}{n_{A}}+\frac{1}{n_{B}}}}
\end{aligned}
$$

Where $\bar{X}_{A}$ and $\bar{X}_{B}$ are the means of the $n_{A}$ and $n_{B}$ performance measurements of instruments A and B. The pooled standard deviation, $s_{p}$ is calculated according to Equation (5.5).

$$
s_{p}=\sqrt{\frac{\left(n_{A}-1\right) s_{A}^{2}+\left(n_{B}-1\right) s_{B}^{2}}{n_{A}+n_{B}-2}}
$$

Where $s_{A}$ and $s_{B}$ are the standard deviations of the of the $n_{A}$ and $n_{B}$ performance measurements of instruments A and B.

To ascertain whether we are to reject each of the null hypothesis in Equations (5.1) and (5.2), we compare the test statistics of Equations (5.3) and (5.4) to an associated rejection criteria. To define this rejection criteria, we must determine the largest probability of committing a type I error that we are willing to accept. Recall that a type I error occurs when we erroneously reject the null hypothesis. In the equivalence test setting, a type I error means that we conclude that instruments' performances are equivalent when in fact they are truly different. See Leber, Pibida, and Enders (2019) for a discussion on selecting an acceptable type I error probability in the context of radiation and nuclear detection systems. We denote the probability of committing a type I error with the Greek letter $\alpha$.

With $\alpha$ identified, and $T_{L}$ and $T_{U}$ calculated according to Equations (5.3) and (5.4), we reject the null hypothesis of Equation (5.1) if:

$$
T_{L}>t_{1-\alpha, n_{A}+n_{B}-2}
$$

and we reject the null hypothesis of Equation (5.2) if:

$$
T_{U}<-t_{1-\alpha, n_{A}+n_{B}-2}
$$

Here, $t_{1-\alpha, n_{A}+n_{B}-2}$ is the $(1-\alpha) \times 100$ th Student's $t$ quantile with $n_{A}+n_{B}-2$ degrees of freedom. Student's $t$ quantile values are easily assessable through tables found in statistics textbooks, e.g., Montgomery and Runger (2018), or lookup functions in statistical software, spreadsheet tools, and online calculators.

If we are able to reject the null hypothesis in both Equations (5.1) and (5.2), then we reject the overall null hypothesis of the equivalence test, and conclude that the instruments' performances are the same. If we fail to reject either of the null hypotheses of Equations (5.1) and (5.2), then we cannot reject the overall null hypothesis of the equivalence test, and thus we are unable to deem that the instruments' performances are the same. 


\subsection{Example}

In the spirit of using the ANSI N42.32 standard as an example, a personal radiation detection (PRD) system is subjected to an over-range shock for photons by subjecting the instrument to a gamma-ray field that exceeds its maximum measurable exposure rate by a factor of two for two minutes. The instrument's pre- and post-shock performance is captured by its ability to measure the radiation exposure rate of a small radioactive source that produces a stable radiation field. The goal is to prove that the instrument was not adversely affected by the over-range shock for photons. That is, we want to show that the instrument's pre-shock performance is equivalent to its postshock performance. We define equivalence as a difference between the pre-shock and post-shock performance that does not exceed $15 \%$ of the pre-shock performance. We will perform this test at the $\alpha=0.05$ level.

In the test area a radiation field of $30 \mu \mathrm{R} / \mathrm{h}\left(7.74 \times 10^{-9}(\mathrm{C} / \mathrm{kg}) / \mathrm{h}\right)$ is produced by a small radioactive source at the reference point of the instrument, the instrument provided the following pre- and post-shock readings:

pre-shock ( $\mu \mathrm{R} / \mathrm{h}): 30,30,30,30,30,31,31,31,31,31$

post-shock ( $\mu \mathrm{R} / \mathrm{h}): 28,30,30,30,29,29,29,30,30,30$

The summary statistics for these observations are:

$$
\bar{x}_{\text {pre }}=30.5 \quad s_{\text {pre }}=0.527 \quad n_{\text {pre }}=10 \quad \bar{x}_{\text {post }}=29.5 \quad s_{\text {post }}=0.707 \quad n_{\text {post }}=10
$$

We first define the lower and upper bounds of the indifference zone, $\left[\delta_{L}, \delta_{U}\right]$, as $\pm 15 \%$ of the preshock performance:

$$
\delta_{L}=-0.15 \bar{x}_{\text {pre }}=-4.575 \mu R / h \quad \text { and } \quad \delta_{U}=0.15 \bar{x}_{\text {pre }}=4.575 \mu R / h .
$$

Next, we calculated the pooled standard deviation, $s_{p}$, according to Equation (5.5) and the test statistics $T_{L}$ and $T_{U}$ according to Equations (5.3) and (5.4).

$$
\begin{aligned}
& s_{p}=\sqrt{\frac{\left(n_{A}-1\right) s_{A}^{2}+\left(n_{B}-1\right) s_{B}^{2}}{n_{A}+n_{B}-2}}=\sqrt{\frac{(10-1) 0.527^{2}+(10-1) 0.707^{2}}{10+10-2}}=0.6236 \\
& T_{L}=\frac{\left(\bar{X}_{A}-\bar{X}_{B}\right)-\delta_{L}}{S_{p} \sqrt{\frac{1}{n_{A}}+\frac{1}{n_{B}}}}=\frac{(30.5-29.5)-(-4.575)}{0.6236 \sqrt{\frac{1}{10}+\frac{1}{10}}}=19.990 \\
& T_{U}=\frac{\left(\bar{X}_{A}-\bar{X}_{B}\right)-\delta_{U}}{S_{p} \sqrt{\frac{1}{n_{A}}+\frac{1}{n_{B}}}}=\frac{(30.5-29.5)-(4.575)}{0.6236 \sqrt{\frac{1}{10}+\frac{1}{10}}}=-12.819
\end{aligned}
$$

And finally, we formulate our conclusion by comparing the test statistics $T_{L}=19.990$ and $T_{U}=-12.819$ with their appropriate rejection regions as defined by Equations (5.6) and (5.7). With $t_{1-\alpha, n_{A}+n_{B}-2}=t_{0.95,18}=1.734$, we reject the null hypothesis of Equation (5.1) since $T_{L}>1.734$ and we 
reject the null hypothesis of Equation (5.2) since $T_{U}<-1.734$. Because we reject the null hypothesis in both Equations (5.1) and (5.2), then we reject the overall null hypothesis of the equivalence test, and conclude that the instrument's post-shock performance is equivalent to its pre-shock performance.

\subsection{Confidence Interval Representation}

In general, a hypothesis test can be formulated and presented in terms of a confidence interval that is compared to some threshold. For the equivalence test presented in this section, we reject the overall null hypothesis of the equivalence test and conclude that the instruments' performances are the same at the $\alpha$-level of significance if the confidence interval provided in Equation (5.8) is entirely contained within the indifference zone $\left[\delta_{L}, \delta_{U}\right]$.

$$
\left(\bar{X}_{A}-\bar{X}_{B}\right) \pm t_{1-\alpha, n_{A}+n_{B}-2} s_{p} \sqrt{\frac{1}{n_{A}}+\frac{1}{n_{B}}}
$$

The confidence interval of Equation (5.8) is formulated by combining the two one-sided confidence intervals that correspond to the tests of Equations (5.1) and (5.2). The result is a $100(1-2 \alpha) \%$ two-sided confidence interval with an expansion factor based on the $100(1-\alpha) t h$ Student's $t$ quantile. Note that this is unlike the $100(1-\alpha) \%$ confidence intervals presented in Section 3 that are used to detect a difference between two instruments and rely on an expansion factor based on the $100\left(1-\frac{\alpha}{2}\right)$ th Student's $t$ quantile.

In continuing with the above example, the confidence interval for equivalence is:

$$
\left(\bar{X}_{A}-\bar{X}_{B}\right) \pm t_{1-\alpha, n_{A}+n_{B}-2} S_{p} \sqrt{\frac{1}{n_{A}}+\frac{1}{n_{B}}}=(30.5-29.5)-1.734 \cdot 0.6236 \sqrt{\frac{1}{10}+\frac{1}{10}}=1 \pm 0.484=[0.516,1.484]
$$

Since this interval is entirely contained within the indifference zone $\left[\delta_{L}, \delta_{U}\right]=[-4.575,4.575]$ we reject the overall null hypothesis of the equivalence test, and conclude that the instrument's postshock performance is equivalent to its pre-shock performance. An illustration of this confidence interval approach is provided in Figure 7.

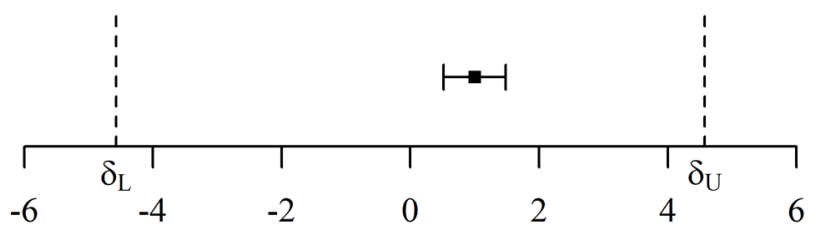

Difference in pre- and post-test response $(\mu \mathrm{R} / \mathrm{h})$

Figure 7: Combined one-sided $95 \%$ confidence intervals for the difference in the pre-and posttest response. The indifference zone $\left[\delta_{\mathrm{L}}, \delta \mathrm{u}\right]$ is displayed by the dashed lines.

\subsection{Power and Sample Size}

As with the traditional formulation of a hypothesis test, two errors are possible in an equivalence test. A type I error occurs when the equivalence test leads us to conclude that the performances of 
the instruments are the same when in fact, they are truly different. While a type II error is when we fail to conclude that the performances of the instruments are equivalent when they are truly the same. We can control the probabilities of these errors through our choice of sample size and can use a power curve to evaluate the expected impact of sample size on the equivalence test's type I and type II errors.

In the case of the equivalence test, the power curve provides the probability of concluding that the instruments are equivalent (i.e., rejecting the null hypothesis) as a function of the true difference in the instruments' performances. Ideally, when the true difference in the instruments' performances is within the indifference zone we would always conclude (probability of one) that the instruments are equivalent and otherwise always fail to conclude (probability of zero) that the instruments are equivalent. This ideal power curve, for the above example with the indifference zone $\left[\delta_{L}, \delta_{U}\right]=[-4.575,4.575]$, is displayed in Figure.

Unfortunately, a test with no risk (no type I and type II errors), such as the ideal test illustrated with the power profile displayed in Figure 8, requires an infinite number of samples. Therefore, common practice is to state a maximum acceptable type I error probability and construct a suitable acceptance criterion and sample size. The resulting power curve is examined, and the sample size adjusted to satisfy the desired type II error probability. See Leber, Pibida, \& Enders(2002) for further discussion on setting maximum acceptable errors in the context of homeland security applications.

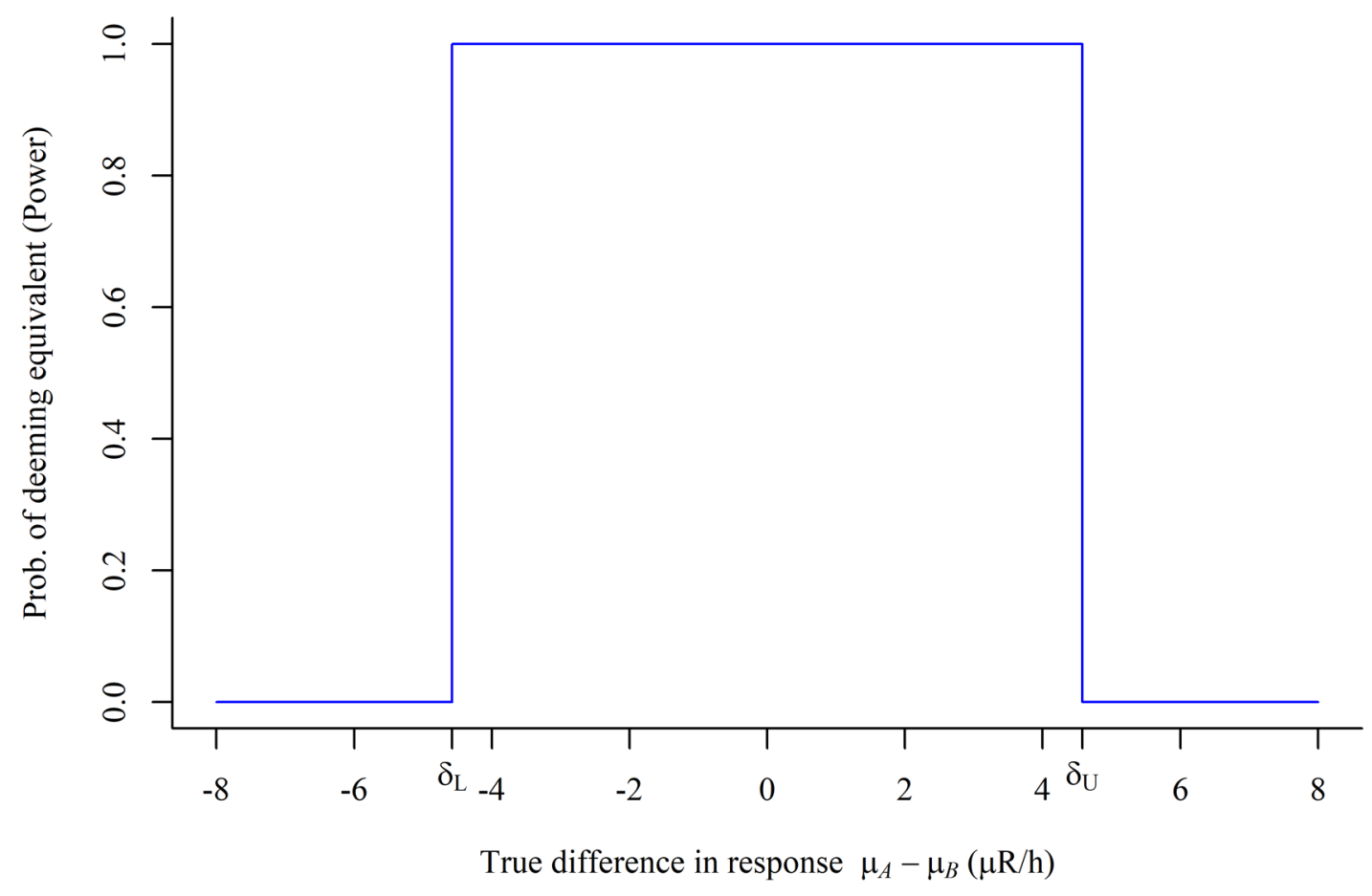

Figure 8: Power curve for an ideal equivalence test with indifference zone

$$
\left[\delta_{\llcorner}, \delta_{U}\right]=[-4.575,4.575]
$$


To calculate the power of the equivalence test for a given finite sample size, $\left\{n_{A}, n_{B}\right\}$, we must calculate the probability of rejecting the null hypotheses of both Equations (5.1) and (5.2). Since we reject the null hypothesis of Equation (5.1) when $T_{L}>t_{1-\alpha, n_{A}+n_{B}-2}$ and we reject the null hypothesis of Equation (5.2) when $T_{U}<-t_{1-\alpha, n_{A}+n_{B}-2}$, then it follows that the power of the equivalence test is provided by Equation (5.9).

$$
\text { Power }=P\left(T_{L}>t_{1-\alpha, n_{A}+n_{B}-2} \text { and } T_{U}<-t_{1-\alpha, n_{A}+n_{B}-2}\right)
$$

where $T_{L}$ and $T_{U}$ are the test statistics provided by Equations (5.3) and (5.4) that are distributed according to a non-central $t$-distribution with non-centrality parameters $\left(\mu_{A}-\mu_{B}-\delta_{L}\right) /\left(\sigma \sqrt{\frac{1}{n_{A}}+\frac{1}{n_{B}}}\right)$ and $\left(\mu_{A}-\mu_{B}-\delta_{U}\right) /\left(\sigma \sqrt{\frac{1}{n_{A}}+\frac{1}{n_{B}}}\right)$, respectively (Leber, Pibida et al. 2019). Using Equation (5.9) and $s_{p}$ as an estimate for $\sigma$, we calculate the power for the test from the above example with $n_{A}=n_{B}=10$ and several alternative sample sizes. These power curves are presented, along with the ideal power curve $\left(n_{A}=n_{B}=\infty\right)$ in Figure 9 .

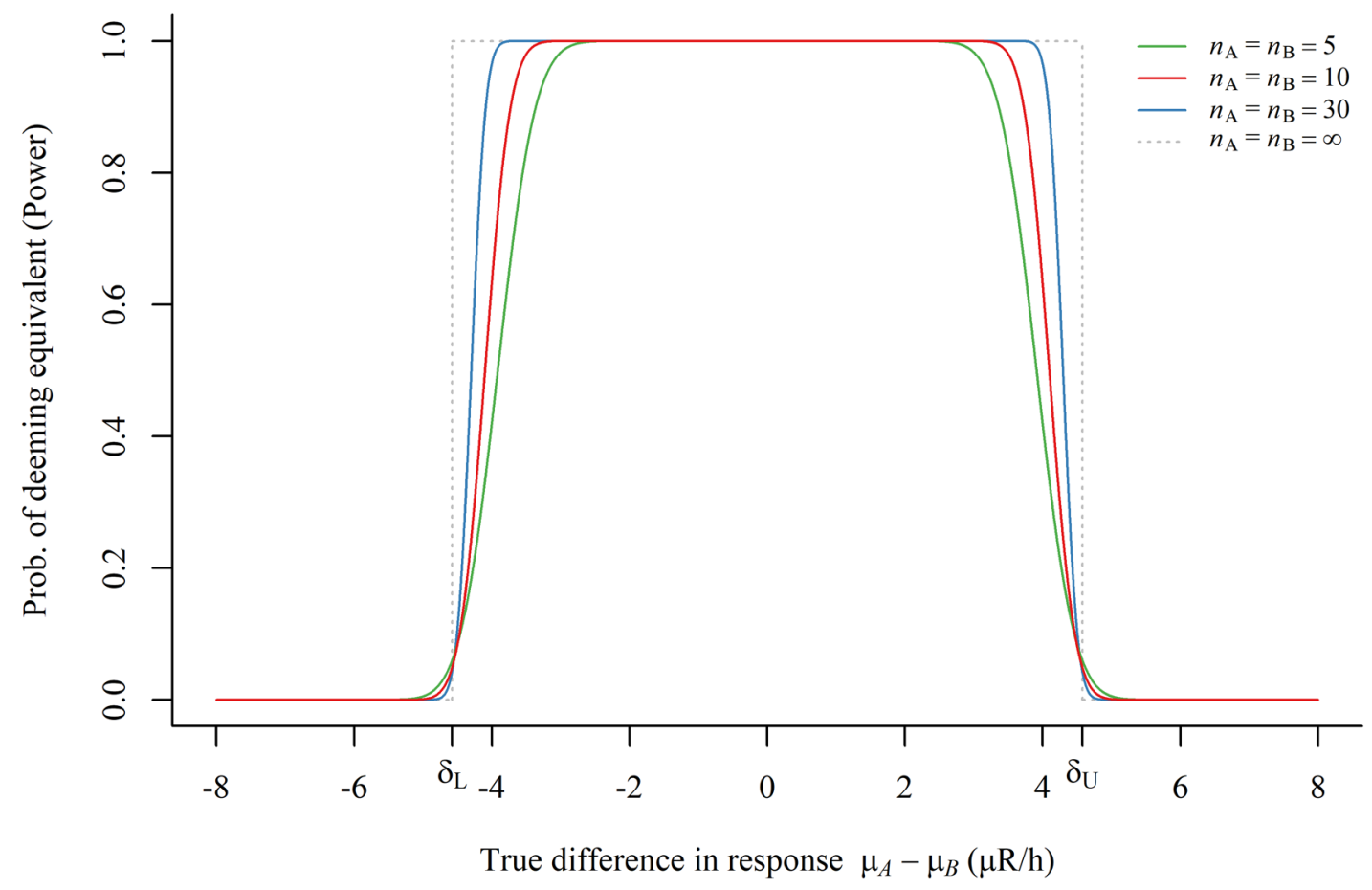

Figure 9: Power curves for several equivalence tests of varying sample sizes, $n_{A}=n_{B}$, each with a maximum type I error probability $\alpha=0.05$, indifference zone $\left[\delta_{L}, \delta_{l}\right]=[-4.575,4.575]$, and $s$ estimated as 0.624 .

Among the finite tests displayed in Figure 9, we observe that the power curve of the $n_{A}=n_{B}=30$ test most closest represents that of the ideal $n_{A}=n_{B}=\infty$ test. Further, when the true difference in 
the instruments' responses is of practical significance to the experimenter, i.e., falls outside of the indifference zone $\left[\delta_{L}, \delta_{U}\right]=[-4.575,4.575]$, the probability of erroneously deeming the instruments equivalent does not exceed 0.05 for all of the finite tests. We also note that for all tests displayed in Figure 9 the power curves reach their maximum value of one within the indifference zone. The difference between the tests is the rate at which they reach the maximum power. Consider, for example, two "equivalent" instruments whose true difference in response is $4 \mu \mathrm{R} / \mathrm{h}\left(1.03 \times 10^{-9}\right.$ $(\mathrm{C} / \mathrm{kg}) / \mathrm{h})$, then, from the curves provided in Figure 9, we observe:

1. When $n_{A}=n_{B}=5$, the probability of deeming these truly equivalent instruments as such is 0.42 (probability of a type II error $=0.58$ );

2. When $n_{A}=n_{B}=10$, the probability of deeming these truly equivalent instruments as such is 0.63 (probability of a type II error $=0.37$ ); and,

3. When $n_{A}=n_{B}=30$, the probability of deeming these truly equivalent instruments as such is 0.97 (probability of a type II error $=0.03$ ).

Thus, amongst the three finite tests illustrated in Figure 9, we are most likely to draw the correct conclusion in the $n_{A}=n_{B}=30$ test. The experimenter must consider whether this increase in test performance (power) is worth the increased cost associated with the additional required samples.

In Figure 10 we illustrate how variability in the test measurements, in addition to the sample size, effects the power of the equivalence test. In Figure 10 we again calculate the power for the test from the above example, however, here we have increased $s_{p}$, the estimate for $\sigma$, by a factor of four. 


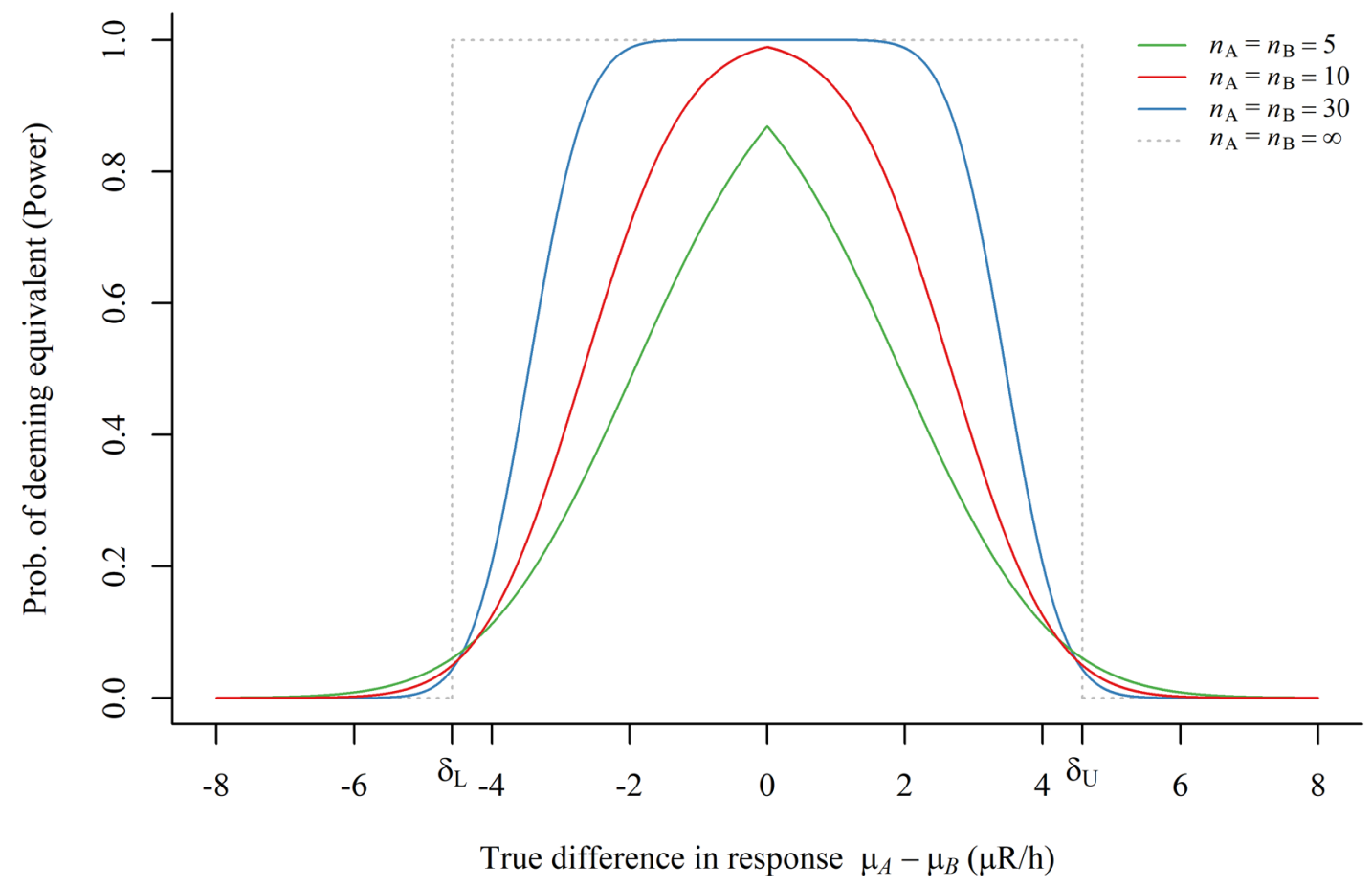

Figure 10: Power curves for several equivalence tests of varying sample sizes, $n_{A}=n_{B}$, each with a maximum type I error probability $a=0.05$, indifference zone $\left[\delta_{\mathrm{L}}, \delta \mathrm{u}\right]=[-4.575,4.575]$, and $s$ estimated as 2.494.

By comparing the power curves in Figure 10 to those in Figure 9, we see that the increased variability in the measurements has reduced our ability to deem truly equivalent systems as such. As an extreme case, consider two instruments whose responses are exactly the same, i.e., the true difference in response is $0 \mu \mathrm{R} / \mathrm{h}(0(\mathrm{C} / \mathrm{kg}) / \mathrm{h})$. With the lower measurement variability of Figure 9 , the probability of deeming these two systems equivalent is one, even with the small sample size of $n_{A}=n_{B}=5$. However, when the measurement variability is larger (Figure 10), we see that for the small sample size test of $n_{A}=n_{B}=5$, the probability of deeming these two systems equivalent is 0.87 . That is, there is a $13 \%$ chance that the test will fail to deem these two systems equivalent.

\subsection{Other Applications}

This section provided an overview of an equivalence test that allows for experimenter to prove that the true mean performances of two (or more) instruments are the same. Pardo (2002) provides practical applications of equivalence tests that expand beyond this application to include the equivalence of proportions and variances. 


\section{References}

[1] Leber, D. D., Pibida, L., \& Enders, A. L. (2019, June). Confirming a Performance Threshold with a Binary Experimental Response. NIST Technical Note. Retrieved from https://doi.org/10.6028/NIST.TN.2045

[2] NIST/SEMATECH e-Handbook of Statistical Methods, available at:

http://www.itl.nist.gov/div898/handbook/

[3] Mendenhall, W, \& Sincich, T. (1992). Statistics for Engineering and the Sciences, Third Edition, Dellen - MacMillan, New York.

[4] Hahn, G. \& Meeker, W. Q. (1991). Statistical Intervals: A Guide for Practitioners, John Wiley \& Sons, Inc., New York.

[5] Ross, S. (1976). A First Course in Probability, Macmillan, New York.

[6] Agresti, A., \& Caffo, B., (2000). Simple and Effective Confidence Intervals for Proportions and Differences of Proportions Result from Adding Two Successes and Two Failures, The American Statistician, 54(4), 280-288.

[7] Fleiss, J. L., (1981). Statistical Methods for Rates and Proportions, Second Edition, John Wiley \& Sons, New York.

[8] Snedecor, G.W. \& Cochran, W.G. (1989). Statistical Methods, Eighth Edition, Iowa State University Press, Ames, IA.

[9] Brown, M.B. \& Forysthe, A.B. (1974), Journal of the American Statistical Association, 69, 364-367.

[10] American National Standards Institute. (2016). American National Standard Performance Criteria for Alarming Personal Radiation Detectors for Homeland Security (N42.32), Institute of Electrical and Electronics Engineers, Inc., New York.

[11] Anderson-Cook, C. M., \& Borror, C. M. (2016). The difference between "equivalent" and "not different". Quality Engineering, 28(3), 249-262.

[12] Casella, G., \& Berger, R. L. (2002). Statistical Inference, Second Edition, Duxbury, Pacific Grove, CA.

[13] Pardo, S. (2014). Equivalence and Noninferiority Tests for Quality, Manufacturing and Test Engineers, CRC Press, Boca Raton, FL.

[14] Richter, S. J., \& Richter, C. (2002). A Method for Determining Equivalence in Industrial Applications. Quality Engineering, 14(3), 375-380.

[15] Wellek, S. (2010). Testing Statistical Hypotheses of Equivalence and Noninferiority, Second Edition, Chapman \& Hall/CRC, Boca Raton, FL. 
[16] Montgomery, D.C.. \& Runger, G.C. (2018). Applied Statistics and Probability for Engineers, Seventh Edition, John Wiley \& Sons, Inc., New York. 